\title{
Recovering Degenerate Kernels in Hyperbolic Integro-Differential Equations
}

\author{
J. Janno and A. Lorenzi
}

\begin{abstract}
The problem of recovering a degenerate operator kernel in a hyperbolic integro-differential operator equation is studied. Existence, uniqueness and stability for the solution are proved. A conditional convergence of a sequence of solutions corresponding to degenerate kernels to a solution corresponding to a non-degenerate kernel is shown. Such results are applied to determine space- and time-dependent relaxation kernels in a multi-dimensional viscoelastic wave equation with given boundary observations of traction type on the assumption that the kernels to be determined are representable as a finite or infinite sum of products of known space-dependent and unknown time-dependent functions.
\end{abstract}

Keywords: Identification problems, hyperbolic integro-differential equations, secondorder integro-differential operator equations, space- and time-dependent degenerate relaxation kernels

AMS subject classification: Primary 45Q05, secondary 45K05, 45N05, 35L20, 34G10, 47D06, 47D09

\section{Introduction}

The problem of recovering unknown kernels in integro-differential evolution equations has been intensively studied during the last decade. Such a problem occurs in applications when describing intrinsic properties of materials with memory. Up to now a thorough treatment has been obtained concerning the identification of time-dependent kernels in hyperbolic equations (see [3, 4, 10] - $[18,26])$ both when the data are exactly known and when they are affected by some (known) error. However, many questions are open in the case the kernels to be determined are both time- and space-dependent. Partial results

J. Janno: Tallinn Techn. Univ., Inst. Cyb., 12618 Tallinn, Estonia; janno@ioc.ee. The author was also supported by the Estonian Science Foundation, Grant Nr. 4706. A. Lorenzi: Univ. degli Studi di Milano, Dept. Math. "F. Enriques", via Saldini 50, 20133 Milano, Italy; lorenzi@mat.unimi.it.

Work partially supported by the Italian Ministero dell'Università e della Ricerca Scientifica e Tecnologica.

ISSN 0232-2064 / \$2.50 C Heldermann Verlag Berlin 
have been obtained when the kernels depend only on some space variables [20, 21] (for the corresponding parabolic case see [5] - [7]) under the assumption that the data are exactly known.

The general case, when the kernel depends on time and all the space variables but the operator under the integral sign is of zero-th order, has been dealt with in $[1,2]$ using as an additional information the so-called Dirichlet-toNeumann map. Of course, in this case, due to the huge overdetermination of the identification problem, only the uniqueness and the continuous dependence of the solution on the data can be derived.

Coming back to problems with exact data, we recall that in [19] a different approach for such problems was proposed, consisting in representing a space- and time-dependent kernel as a sum of products of known spacedependent and unknown time-dependent functions. In the mentioned paper a one-dimensional hyperbolic inverse problem for such kernels admitting a finite representation was studied.

In the present paper, we will first consider the problem of recovering degenerate kernels appearing in a hyperbolic integro-differential equation related to viscoelastic multi-dimensional materials when boundary observations of traction type are available. The kernels to be recovered are assumed to be representable as a finite or infinite sum of products of known space-dependent and unknown time-dependent functions. More explicitly, under suitable assumptions on the data we will prove existence, uniqueness and stability for the solutions of such problems. Then, assuming that the data are exactly known, we will establish that a sequence of solutions corresponding to degenerate kernels can converge to a solution corresponding to a general kernel, i.e a non-degenerate one, only under very severe assumptions on the data and the solution of the non-degenerate problem. This seemingly astonishing occurrence actually depends on the fact that we simply assume the existence of a solution to a non-degenerate problem, but not any uniqueness of it. So, roughly speaking, the failing of a good approximation of non-degenerate kernels depending on time and all the spatial variables by degenerate ones simply exhibits the severe ill-posedness of the problem under consideration.

From now on our aim will consist in determining a general kernel $m$ depending on time and all the space variables. In this context it seems reasonable to fix an orthogonal basis $\left\{\mu_{n}\right\}_{n \in \mathbb{N}}$ in $L^{2}(\Omega)$ and develop $m$ into the Fourier series $m(t, x)=\sum_{n=1}^{+\infty} m_{n}(t) \mu_{n}(x)$, the series converging in $L^{2}(\Omega)$. Consequently, the problem of recovering $m$ turns out to be equivalent to that of determining the sequence $\left\{m_{n}\right\}_{n \in \mathbb{N}}$ of its Fourier coefficients, i.e. a sequence of functions depending on time only. Therefore, from a mathematical point of view, we are forced to provide a sequence of additional measurements $\left\{\Psi_{n}\right\}_{n \in \mathbb{N}}$, say of traction type. Unfortunately, although this choice is quite reasonable from a physical point of view, on the contrary it is strongly 
singular (cf. Section 8) from a mathematical point of view so that neither any reconstruction of the sequence $\left\{m_{n}\right\}_{n \in \mathbb{N}}$ can be obtained nor any stable approximation of the identification problem with a general kernel $m$ can be constructed, unless, as we said above, the data and the solution corresponding to the general case satisfy severe regularity assumptions.

We conclude this section by giving the plan of the paper.

In Section 2 we pose an inverse hyperbolic identification problem occurring in viscoelasticity and in Section 3 we reformulate this problem in an abstract form. Section 4 collects some information concerning cosine families. In Section 5 the abstract problem is transformed into a more easy-to-handle (equivalent) one, which is solved in Section 6. The problem of dealing with a general operator kernel is dealt with in Section 7. Section 8 contains an application of the abstract results of Sections $3-7$ to the problem in viscoelasticity. Finally, Section 9 is devoted to the proof of a continuous dependence result.

\section{Formulation of a problem related to non-homoneneous viscoelastic materials}

Let $\Omega$ be an $l$-dimensional bounded open set filled by a non-homogeneous viscoelastic material of Boltzmann type. In many one- and two-dimensional cases, but also in some very special three-dimensional configurations, the displacement $u$ and the stress $\sigma$ of a material point $x$ of the body at time $t$ satisfy the constitutive law

$$
\sigma(t, x)=-\lambda(x) \nabla u(t, x)+\int_{-\infty}^{t} m(t-\tau, x) \nabla u(\tau, x) d \tau
$$

for $x \in \Omega$ and $t \in \mathbb{R}$ where $\lambda$ is the instantaneous stress modulus and $m$ is the relaxation kernel. In what follows we assume that the kernel $m$ is of the form:

$$
m(t, x)=\sum_{k=1}^{N} m_{k}(t) \mu_{k}(x) \quad(N \in \mathbb{N} \cup\{+\infty\})
$$

where $\mu_{k}$ are given functions, while $m_{k}$ are unknown, and $\mathbb{N}$ is the set of positive integers. In some applications the kernel $m$ is degenerate, i.e. it can be represented by a finite sum (2.2). This is the case, for instance, when the body $\Omega$ consists of a finite number of homogeneous pieces $\Omega_{k} \quad(k=1, \ldots, N ; N<$ $+\infty)$. Moreover, we can take $m$ of form (2.2) with $\mu_{k}$ being a suitable smooth approximation of the characteristic function of $\Omega_{k}$. In more general cases we have to deal with a problem with $N=+\infty$ so that the corresponding problems with $N<+\infty$ can be considered as natural discretizations of the continuous one. 
Inserting (2.1) - (2.2) into the balance equation

$$
D_{t}^{2} u(t, x)+\operatorname{div} \sigma(t, x)=f(t, x)
$$

and assuming $u(t, x)=0$ for $t<0$, we derive the hyperbolic equation

$$
\begin{aligned}
D_{t}^{2} u(t, x)= & \operatorname{div}(\lambda(x) \nabla u(t, x)) \\
& -\sum_{k=1}^{N} \int_{0}^{t} m_{k}(t-\tau) \operatorname{div}\left(\mu_{k}(x) \nabla u(\tau, x)\right) d \tau+f(t, x)
\end{aligned}
$$

for $x \in \Omega$ and $t \in(0, T)$. To this equation we add the initial and boundary conditions

$$
\left.\begin{array}{rl}
u(0, x) & =\varphi(x) \\
D_{t} u(0, x) & =\psi(x)
\end{array}\right\} \quad(x \in \Omega)
$$

and

$$
\left.u(t, x)\right|_{x \in \Gamma}=\left.\hat{u}(t, x)\right|_{x \in \Gamma},
$$

respectively, where $\Gamma$ is the boundary of the body $\Omega$. Let us suppose that, in addition to the function $u$, the vector of kernels $m=\left(m_{k}\right)_{k=1, \ldots, N}$ is also unknown. In order to recover both $u$ and $m$ we need to complement relations (2.3) - (2.5) with $N$ additional conditions. For instance, when $l \geq 2$, these conditions may be given in the form of $N$ measurements of the traction over the boundary $\Gamma$

$$
\left\langle\eta_{i}, \nu \cdot \sigma(t, \cdot)\right\rangle_{1 / 2, \Gamma}=h_{i}(t)
$$

for $t \in(0, T)$ and $i=1, \ldots, N$. Here $\langle\cdot, \cdot\rangle_{1 / 2, \Gamma}$ denotes the pairing between $H^{-1 / 2}(\Gamma)$ and $H^{1 / 2}(\Gamma)$, the $\eta_{i}$ 's belong to $H^{-1 / 2}(\Gamma)$ and represent the weights related to the $N$ measurements, and $\nu$ stands for the outer normal vector to $\Gamma$.

Remark 2.1. In practice we can assume that each support $\operatorname{supp} \eta_{i}$ is contained in an open subset $\Gamma_{i}$ of $\Gamma$, the $\Gamma_{i}$ 's being pairwise disjoint.

In the one-dimensional case $l=1$ let us take $\Omega=(0,1)$. As additional conditions we can choose $N$ measurements of the stress in different points $x_{i}$ of the interval $[0,1]$ as $\sigma\left(t, x_{i}\right)=g_{i}(t)$ for $t \in(0, T)$ and $i=1, \ldots, N$. For technical reasons we will deal with these relations in the slightly different form

$$
\eta_{i} \sigma\left(t, x_{i}\right)=h_{i}(t)
$$

for $t \in(0, T)$ and $i=1, \ldots, N$ where $\eta_{i} \in \mathbb{R}$ are some properly chosen weights. Inserting (2.1) into (2.6) - (2.7) and taking (2.2) into account, we get the $N$ additional conditions

$$
\Psi_{i}[u(t, \cdot)]-\sum_{i=1}^{N} \int_{0}^{t} m_{k}(t-\tau) \Phi_{i k}[u(\tau, \cdot)] d \tau=h_{i}(t)
$$


for $t \in(0, T)$ and $i=1, \ldots, N$ where

$$
\begin{gathered}
\Psi_{i}[v]= \begin{cases}\left\langle\eta_{i}, \lambda D_{\nu} v\right\rangle_{1 / 2, \Gamma} & \text { if } l \geq 2 \\
\eta_{i} \lambda\left(x_{i}\right) D_{x} v\left(x_{i}\right) & \text { if } l=1\end{cases} \\
\Phi_{i k}[v]= \begin{cases}\left\langle\eta_{i}, \mu_{k} D_{\nu} v\right\rangle_{1 / 2, \Gamma} & \text { if } l \geq 2 \\
\eta_{i} \mu_{k}\left(x_{i}\right) D_{x} v\left(x_{i}\right) & \text { if } l=1 .\end{cases}
\end{gathered}
$$

We now pose our identification problem consisting in determining the pair $(m, u)=\left(\left(m_{k}\right)_{k=1, \ldots, N}, u\right)$ from relations $(2.3)-(2.5),(2.8)$. If we introduce the new unknown

$$
\widetilde{u}=u-\hat{u}
$$

we easily derive the following problem for $(m, \widetilde{u})$ with homogeneous boundary condition

$$
\left.\begin{array}{l}
D_{t}^{2} \widetilde{u}(t, x)=\operatorname{div}(\lambda(x) \nabla \widetilde{u}(t, x)) \\
\quad-\sum_{k=1}^{N} \int_{0}^{t} m_{k}(t-\tau)\left(\operatorname{div}\left(\mu_{k}(x) \nabla \widetilde{u}(\tau, x)\right)+w_{k}(\tau)\right) d \tau+\widetilde{f}(t, x) \\
\widetilde{u}(0, x)=\widetilde{\varphi}(x), D_{t} \widetilde{u}(0, x)=\widetilde{\psi}(x) \\
\left.\widetilde{u}(t, x)\right|_{x \in \Gamma} \equiv 0 \\
\Psi_{i}[\widetilde{u}(t, \cdot)]-\sum_{k=1}^{N} \int_{0}^{t} m_{k}(t-\tau)\left(\Phi_{i k}[\widetilde{u}(\tau, \cdot)]+\chi_{i k}(\tau)\right) d \tau=\widetilde{h}_{i}(t)
\end{array}\right\}
$$

for $t \in(0, T), x \in \Omega$ and $i=1, \ldots, N$ where

$$
\left.\begin{array}{rl}
\widetilde{\varphi}(x) & =\varphi(x)-\hat{u}(0, x), \quad \widetilde{\psi}=\psi-D_{t} \hat{u}(0, x) \\
\widetilde{f}(t, x) & =f(t, x)+\operatorname{div}(\lambda(x) \nabla \hat{u}(t, x))-D_{t}^{2} \hat{u}(t, x) \\
w_{k}(t, x) & =\operatorname{div}\left(\mu_{k}(x) \nabla \hat{u}(t, x)\right) \\
\chi_{i k}(t) & =\Phi_{i k}[\hat{u}(t, \cdot)], \quad \widetilde{h}_{i}(t)=h_{i}(t)-\Psi_{i}[\hat{u}(t, \cdot)]
\end{array}\right\}
$$

for $k=1, \ldots, N$. 


\section{Formulation of an abstract problem}

In this section we reformulate the identification problem (2.11) in an abstract Banach space setting. For this, let $X$ and $Y$ be real Banach spaces, $Y$ being densely embedded into $X$, and let $A$ be a closed linear unbounded operator in $X$ with $D(A)=Y$. We equip $Y$ with the graph norm

$$
\|y\|_{Y}=\|y\|_{X}+\|A y\|_{X} \quad(y \in Y)
$$

where $\|y\|_{X}$ stands for the norm of $y$ in $X$. Let $N \in \mathbb{N} \cup\{+\infty\}$ and assume that for $i, k=1, \ldots, N$

$$
\begin{aligned}
B_{k} & \in \mathcal{L}(Y, X) \\
\Psi_{i}, \Phi_{i k} & \in Y^{\star}
\end{aligned}
$$

where $Y^{\star}$ and $\mathcal{L}(Y, X)$ denote the space dual to $Y$ and the Banach space of all linear bounded operators from $Y$ to $X$, respectively. In particular, we set $\mathcal{L}(X)=\mathcal{L}(X, X)$.

We pose the following

Identification problem. Given $T \in(0,+\infty)$ and, for $i, k=1, \ldots, N$,

$$
\varphi, \psi \in Y, \quad f, w_{k}:(0, T) \rightarrow X, \quad h_{i}, \chi_{i k}:(0, T) \rightarrow \mathbb{R},
$$

find

$$
m_{k}:(0, T) \rightarrow \mathbb{R}, \quad u:(0, T) \rightarrow Y
$$

satisfying for $t \in(0, T)$ and $i=1, \ldots, N$ the equations

$$
\left.\begin{array}{l}
u^{\prime \prime}(t)=A u(t)-\sum_{k=1}^{N} m_{k} *\left(B_{k} u(t)+w_{k}(t)\right)+f(t) \\
u(0)=\varphi, u^{\prime}(0)=\psi \\
\Psi_{i}[u(t)]-\sum_{k=1}^{N} m_{k} *\left(\Phi_{i k}[u(t)]+\chi_{i k}(t)\right)=h_{i}(t)
\end{array}\right\}
$$

where $*$ stands for the convolution operator $\left(v_{1} * v_{2}\right)(t)=\int_{0}^{t} v_{1}(t-\tau) v_{2}(\tau) d \tau$.

In the sequel we will use the affine operators $\widetilde{B}_{k}$ and $\widetilde{\Phi}_{i k}$ defined by the formulae

$$
\left.\begin{array}{rl}
\left(\widetilde{B}_{k} u\right)(t) & =B_{k} u(t)+w_{k}(t) \\
\left(\widetilde{\Phi}_{i k} u\right)(t) & =\Phi_{i k}[u(t)]+\chi_{i k}(t)
\end{array}\right\}
$$

for $t \in(0, T)$ and $i, k=1, \ldots, N$. 


\section{The cosine family}

Due to the simple relation

$$
\left(\widetilde{B}_{k} u\right)(t)=t *\left(\widetilde{B}_{k} u\right)^{\prime \prime}(t)+t\left(\widetilde{B}_{k} u\right)^{\prime}(0)+\left(\widetilde{B}_{k} u\right)(0)
$$

assuming that $w_{k}(k=1, \ldots, N)$ are twice differentiable, Cauchy problem $(3.3)_{1-2}$ for any $u$ such that $u^{\prime \prime}(t) \in Y$ for $t \in(0, T)$ has the equivalent form

$$
\begin{aligned}
u^{\prime \prime}(t)= & t * A u^{\prime \prime}(t) \\
& -\sum_{k=1}^{N} m_{k} *\left[t *\left(\widetilde{B}_{k} u\right)^{\prime \prime}(t)+t\left(\widetilde{B}_{k} u\right)^{\prime}(0)+\left(\widetilde{B}_{k} u\right)(0)\right] \\
& +f(t)+t A \psi+A \varphi \quad(t \in(0, T)) \\
u(0)= & \varphi, u^{\prime}(0)=\psi
\end{aligned}
$$

Let us now consider the operator convolution equation with the same principal part as $(4.1)_{1}$

$$
v(t)=t * A v(t)+g(t) \quad(t \in(0, T))
$$

where $g:(0, T) \rightarrow X$ is a given function. A function $v \in C([0, T] ; Y)$ satisfying this equation is called a strong solution of (4.2).

Next we introduce a resolvent family for equation (4.2), called the cosine family. Let us assume that for the operator $A$ there exist two constants $M \geq 1$ and $\omega \in \mathbb{R}$ such that

1) $\lambda^{2} \in \rho(A)$ for all $\lambda>\omega$ where $\rho(A)$ is the resolvent set of $A$ $n \in \mathbb{N}$.

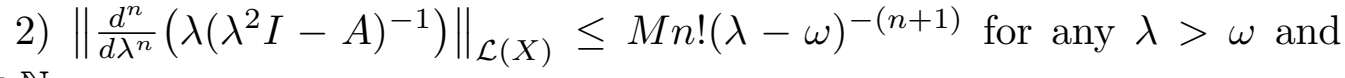
Such an assumption is necessary and sufficient for $A$ to generate a cosine family, i.e. a family of operators $\{\mathcal{C}(t)\}_{t \in \mathbb{R}} \subset \mathcal{L}(X)$ satisfying the following conditions (see [9: Chapter 2] and [24: Section 1.1/Theorem 1.3]):

$$
\left.\begin{array}{l}
\mathcal{C}(t) \text { strongly continuous on } \mathbb{R}, \mathcal{C}(0)=I \\
\mathcal{C}(t+s)+\mathcal{C}(t-s)=2 \mathcal{C}(t) \mathcal{C}(s) \quad(t, s \in \mathbb{R}) \\
\mathcal{C}(t) Y \subset Y \text { and } A \mathcal{C}(t) y=\mathcal{C}(t) A y \quad(y \in Y, t \in \mathbb{R}) \\
\mathcal{C}(t) y=y+t * A \mathcal{C}(t) y \quad(y \in Y, t \in \mathbb{R}) \\
A=\mathcal{C}^{\prime \prime}(0)
\end{array}\right\}
$$

The cosine family, if it exists, is unique. 
By means of $\mathcal{C}(t)$ we can also define the interpolation space $Y_{1}$ associated with $X$ and $Y$ by

$$
Y_{1}=\left\{x \in X: t \rightarrow \mathcal{C}^{\prime}(t) x \in C([0, T] ; X) \text { for any } t \in \mathbb{R}\right\} .
$$

The space $Y_{1}$ is equipped with the norm (see [25])

$$
\|x\|_{Y_{1}}=\|x\|_{X}+\sup _{0 \leq t \leq 1}\left\|\mathcal{C}^{\prime}(t) x\right\|_{X}
$$

Let us prove the following

Lemma 4.1. Let $A$ generate a cosine family in $X$. Then the following propositions hold:

(i) $\mathcal{C}(t) \in \mathcal{L}(Y), 1 * \mathcal{C}(t) \in \mathcal{L}\left(Y_{1}, Y\right)$ and $t * \mathcal{C}(t) \in \mathcal{L}(X, Y)$ for any $t \in \mathbb{R}$.

(ii) $t \rightarrow \mathcal{C}(t) x \in C([0, T] ; Y)$ for any $x \in Y$.

(iii) $t \rightarrow 1 * \mathcal{C}(t) x \in C([0, T] ; Y)$ for any $x \in Y_{1}$.

(iv) $t \rightarrow t * \mathcal{C}(t) x \in C([0, T] ; Y)$ for any $x \in X$.

Proof. The assertions $\mathcal{C}(t) \in \mathcal{L}(Y)$ for $t \in \mathbb{R}$ and assertion (ii) easily follow from properties $(4.3)_{3},(4.3)_{4}$ and the definition of the norm $\|\cdot\|_{Y}$. The assertions $t * \mathcal{C}(t) \in \mathcal{L}(X, Y)$ for $t \in \mathbb{R}$ and assertion (iv) are direct consequences of [24: Proposition 1.1].

In order to prove that $1 * \mathcal{C}(t) \in \mathcal{L}\left(Y_{1}, Y\right)$ we first note that, due to the definitions of $Y_{1}$ and the norm $\|\cdot\|_{Y_{1}}$, we immediately have

$$
\mathcal{C}^{\prime}(t) \in \mathcal{L}\left(Y_{1}, X\right)
$$

for any $t \in[0,1]$. This relation may be extended to arbitrary $t \in \mathbb{R}$ using the equalities $\mathcal{C}^{\prime}(2 t)=2 \mathcal{C}(t) \mathcal{C}^{\prime}(t)$ and $\mathcal{C}^{\prime}(-t)=-\mathcal{C}^{\prime}(t)$, implied by $(4.3)_{2}$, and the property $\mathcal{C}(t) \in \mathcal{L}(X) \quad(t \in \mathbb{R})$.

Next we use the formula

$$
\mathcal{C}(s)[1 * \mathcal{C}(t)]=\frac{1}{2} \int_{0}^{t} \mathcal{C}(\tau+s) d \tau+\frac{1}{2} \int_{0}^{t} \mathcal{C}(\tau-s) d \tau
$$

following from $(4.3)_{2}$. Computing the second derivative of this relation with respect to $s$ at $s=0$ and observing that $\mathcal{C}^{\prime \prime}(0)=A$, we obtain

$$
A[1 * \mathcal{C}(t)]=\frac{1}{2} \mathcal{C}^{\prime}(t)+\frac{1}{2} \mathcal{C}^{\prime}(-t)
$$

This relation by virtue of (4.6) and the definition of the norm in $D(A)=Y$ proves the desired assertion $1 * \mathcal{C}(t) \in \mathcal{L}\left(Y_{1}, Y\right)$. Finally, assertion (iii) follows from (4.7) due to the continuity of $\mathcal{C}^{\prime}(t) x$ in $X$ with respect to $t$ for any $t \in \mathbb{R}$ and $x \in Y_{1}$ (see definition (4.4) of $Y_{1}$ ) 
Let us now formulate and prove a lemma concerning the existence and uniqueness of the solution to equation (4.2).

Lemma 4.2. Let A generate a cosine family in $X$. Then the following assertions hold:

(i) If $v$ is a strong solution of $(4.2)$ and $g \in W^{1,1}((0, T) ; X)$, then

$$
v(t)=\mathcal{C}(t) g(0)+\mathcal{C} * g^{\prime}(t) \quad \text { for a.e. } t \in(0, T) .
$$

In particular, strong solutions are unique.

(ii) Conversely, if $g=g_{1}+1 * g_{2}+t * g_{3}$, where $g_{1} \in W^{1,1}((0, T) ; Y), g_{2} \in$ $W^{1,1}\left((0, T) ; Y_{1}\right)$ and $g_{3} \in W^{1,1}((0, T) ; X)$, then (4.2) has a strong solution $v$ of form (4.8).

Proof. Assertions (i) and (ii) in the case $g_{2}=0$ follow from [24: Proposition 1.2]. It remains to prove assertion (ii) in the case $g_{1}=g_{3}=0$ and $g_{2} \neq 0$. Then function (4.8) has the form $v(t)=1 * \mathcal{C}(t) g_{2}(0)+1 * \mathcal{C} * g_{2}^{\prime}(t)$. By virtue of the properties of $g_{2}$, Lemma $4.1 /$ (iii) and the inclusion $1 * \mathcal{C} \in$ $L^{\infty}\left((0, T) ; \mathcal{L}\left(Y_{1}, Y\right)\right)$, implied by assertions (iii) and $1 * \mathcal{C}(t) \in \mathcal{L}\left(Y_{1}, Y\right) \quad(t \in$ $\mathbb{R})$ of Lemma 4.1 and the uniform boundedness principle, we deduce that $v \in C([0, T] ; Y)$. Now we can immediately check that $v$ is a strong solution of (4.2) inserting it into (4.2) and taking advantage of resolvent equation $(4.3)_{4}$

\section{Reduction to a system of equations of the second kind}

In this section we formulate and prove a lemma ensuring the equivalence of identification problem (3.3) with a system of integral equations for $u^{\prime \prime},\left(m_{k}^{\prime}\right)_{k=1, \ldots, N}$. In the next section such a system will be rewritten in a fixed-point form to which the contraction mapping principle will be applied.

Before stating our lemma, let us introduce some further notation. We set

$$
\begin{array}{lll}
B=\left(B_{k}\right)_{k=1, \ldots, N} & w=\left(w_{k}\right)_{k=1, \ldots, N} & \Psi=\left(\Psi_{i}\right)_{i=1 \ldots, N} \\
m=\left(m_{k}\right)_{k=1, \ldots, N} & \Phi=\left(\Phi_{i k}\right)_{i, k=1 \ldots, N} & \chi=\left(\chi_{i k}\right)_{i, k=1 \ldots, N}
\end{array}
$$

and associate with any Banach space $\mathcal{X}$ the product Banach space

$$
\mathcal{X}^{N}=\left\{x=\left(x_{i}\right)_{i=1, \ldots, N}: x_{i} \in \mathcal{X},\|x\|_{\mathcal{X}^{N}}=\left(\sum_{i=1}^{N}\left\|x_{i}\right\|_{\mathcal{X}}^{2}\right)^{1 / 2}<+\infty\right\} .
$$

Observe that $\mathbb{R}^{\infty}$ coincides with $l_{2}$ in this notation.

Our aim is to seek for the solution $(m, u)$ of $(3.3)$ in the space

$$
\mathcal{S}^{N}=H^{1}\left((0, T) ; \mathbb{R}^{N}\right) \times C^{2}([0, T] ; Y) .
$$


Lemma 5.1. Let $N \in \mathbb{N} \cup\{+\infty\}$ and $T \in(0,+\infty)$. Assume that the operator $A$ generates the cosine family $\{\mathcal{C}(t)\}_{t \in \mathbb{R}}$ in $X$. Moreover, in addition to (3.1) - (3.2), let the following assumptions hold:

$$
\begin{aligned}
& \varphi, \psi \in Y \\
& f=f_{1}+1 * f_{2}+t * f_{3} \text { where }\left\{\begin{array}{l}
f_{1}+A \varphi \in W^{1,1}((0, T) ; Y) \\
f_{2}+A \psi \in W^{1,1}\left((0, T) ; Y_{1}\right) \\
f_{3} \in W^{1,1}((0, T) ; X)
\end{array}\right. \\
& w \in C^{2}\left([0, T] ; X^{N}\right), B \varphi+w(0) \in\left(Y_{1}\right)^{N} \\
& \chi \in C^{2}\left([0, T] ; \mathcal{L}\left(\mathbb{R}^{N}, \mathbb{R}^{N}\right)\right) \\
& h \in H^{2}\left((0, T) ; \mathbb{R}^{N}\right), h(0)=\Psi[\varphi]
\end{aligned}
$$

In the case $N=+\infty$ we also assume

$$
\left.\begin{array}{rl}
B & \in(\mathcal{L}(Y, X))^{\infty} \\
\Psi & \in\left(Y^{\star}\right)^{\infty} \\
\left(\left\|\Phi_{i k}\right\|_{Y^{\star}}\right)_{i, k=1 ; \infty} & \in \mathcal{L}\left(\mathbb{R}^{\infty}, \mathbb{R}^{\infty}\right)
\end{array}\right\} .
$$

Then identification problem (3.3) for the pair $(m, u)$ in $\mathcal{S}^{N}$ is equivalent to the system of equations of the second kind

$$
\begin{aligned}
& u^{\prime \prime}(t)=-\sum_{k=1}^{N}\left[m_{k}(0)+m_{k}^{\prime} *\right] \\
& \times\left[t * \mathcal{C} *\left(\widetilde{B}_{k} u\right)^{\prime \prime}(t)+t * \mathcal{C}(t)\left(\widetilde{B}_{k} u\right)^{\prime}(0)+1 * \mathcal{C}(t)\left(\widetilde{B}_{k} u\right)(0)\right]+\alpha(t) \\
& u(0)=\varphi, u^{\prime}(0)=\psi \\
& \sum_{k=1}^{N} m_{k}^{\prime}(t)\left(\widetilde{\Phi}_{i k} u\right)(0)+\sum_{k=1}^{N} m_{k}^{\prime} * 1 *\left(\widetilde{\Phi}_{i k} u\right)^{\prime \prime}(t) \\
& +\sum_{k=1}^{N} m_{k}^{\prime} *\left(\widetilde{\Phi}_{i k} u\right)^{\prime}(0)+\sum_{k=1}^{N} m_{k}(0) *\left(\widetilde{\Phi}_{i k} u\right)^{\prime \prime}(t)-\Psi_{i}\left[u^{\prime \prime}(t)\right] \\
& =-h_{i}^{\prime \prime}(t)-\sum_{k=1}^{N} m_{k}(0)\left(\widetilde{\Phi}_{i k} u\right)^{\prime}(0) \\
& \sum_{k=1}^{N} m_{k}(0)\left(\widetilde{\Phi}_{i k} u\right)(0)=\Psi_{i}[\psi]-h_{i}^{\prime}(0)
\end{aligned}
$$

for $t \in(0, T)$ and $i=1, \ldots, N$ where

$$
\begin{aligned}
\alpha(t)= & \mathcal{C}(t)\left(f_{1}(0)+A \varphi\right)+\mathcal{C} * f_{1}^{\prime}(t)+1 * \mathcal{C}(t)\left(f_{2}(0)+A \psi\right) \\
& +1 * \mathcal{C} * f_{2}^{\prime}(t)+t * \mathcal{C}(t) f_{3}(0)+t * \mathcal{C} * f_{3}^{\prime}(t)
\end{aligned}
$$


Proof. First, let us prove the assertion of Lemma 5.1 in the case $(5.3)_{1}$ is replaced by equation (4.1) $)_{1}$. At the beginning of Section 3 we showed that Cauchy problem $(3.3)_{1-2}$ is equivalent to (4.1). Since $(4.1)_{2}$ and $(5.3)_{2}$ coincide, problem $(3.3)_{1-2}$ is equivalent to $(4.1)_{1},(5.3)_{2}$. Let us now show that, under initial conditions $(5.3)_{2}$ for $u$, equations $(3.3)_{3}$ are equivalent to subsystem $(5.3)_{3-4}$. This would prove the assertion of Lemma 5.1 in the case $(5.3)_{1}$ is replaced by $(4.1)_{1}$.

We begin by showing implication $(3.3)_{3} \Rightarrow(5.3)_{3-4}$. Let $m \in H^{1}\left((0, T) ; \mathbb{R}^{N}\right)$ solve $(3.3)_{3}$ and assume that $u$ is a function from $C^{2}([0, T] ; Y)$ satisfying $(5.3)_{2}$. Note that, due to our assumptions and the membership $m \in H^{1}\left((0, T) ; \mathbb{R}^{N}\right)$, we can differentiate equation $(3.3)_{3}$ twice and bring the derivatives into the sum. Particularly, in the case $N=+\infty$ the series

$$
\sum_{k=1}^{+\infty} \frac{d^{j}}{d t^{j}}\left[m_{k} *\left(\Phi_{i k}[u(t)]+\chi_{i k}(t)\right)\right] \quad(j=1,2)
$$

converges in $L^{1}(0, T)$. Thus, the first and second derivatives of the sum in $(3.3)_{3}$ exist in the distributional sense. Differentiating $(3.3)_{3}$ and using notation (3.4) we obtain

$$
\Psi_{i}\left[u^{\prime}(t)\right]-\sum_{k=1}^{N} m_{k}(0)\left(\widetilde{\Phi}_{i k} u\right)(t)-\sum_{k=1}^{N} m_{k}^{\prime} *\left(\widetilde{\Phi}_{i k} u\right)(t)=h_{i}^{\prime}(t)
$$

for $t \in(0, T)$ and $i=1, \ldots, N$. Setting $t=0$ in this equation and using $(5.3)_{2}$, we derive $(5.3)_{4}$. Differentiating (5.5) once again, we have

$$
\begin{gathered}
\Psi_{i}\left[u^{\prime \prime}(t)\right]-\sum_{k=1}^{N} m_{k}(0)\left(\widetilde{\Phi}_{i k} u\right)^{\prime}(t) \\
-\sum_{k=1}^{N} m_{k}^{\prime}(t)\left(\widetilde{\Phi}_{i k} u\right)(0)-\sum_{k=1}^{N} m_{k}^{\prime} *\left(\widetilde{\Phi}_{i k} u\right)^{\prime}(t)=h_{i}^{\prime \prime}(t)
\end{gathered}
$$

for $t \in(0, T)$ and $i=1, \ldots, N$. Substituting here $1 *\left(\widetilde{\Phi}_{i k} u\right)^{\prime \prime}(t)+\left(\widetilde{\Phi}_{i k} u\right)^{\prime}(0)$ for $\left(\widetilde{\Phi}_{i k} u\right)^{\prime}(t)$, we derive $(5.3)_{3}$, too. Thus, we have shown that $(3.3)_{3}$ implies $(5.3)_{3-4}$.

Conversely, if (5.3) $)_{3-4}$ hold, then $(3.3)_{3}$ does, too. To show this, we perform the above operations in the opposite order. We substitute $\left(\widetilde{\Phi}_{i k} u\right)^{\prime}(t)-$ $\left(\widetilde{\Phi}_{i k} u\right)^{\prime}(0)$ for $1 *\left(\widetilde{\Phi}_{i k} u\right)^{\prime \prime}(t)$ in $(5.3)_{3}$ and get (5.6). Thereupon, after integrating both sides in $(5.6)$ over $[0, t]$, from the initial condition $u^{\prime}(0)=\psi$ and relations $(5.3)_{4}$ we deduce (5.5). Finally, integrating both sides in (5.5) 
again over $[0, t]$ and using the initial condition $u(0)=\varphi$ and $(5.1)_{6}$, we obtain $(3.3)_{3}$. Summing up, we have proved the assertion of Lemma 5.1 when $(5.3)_{1}$ is replaced by $(4.1)_{1}$.

It remains to show the equivalence of $(4.1)_{1}$ and $(5.3)_{1}$ if $u^{\prime \prime} \in C([0, T] ; Y)$. Noting that $f(t)=f_{1}(t)+1 * f_{2}(t)+t * f_{3}(t)$, we can rewrite $(4.1)_{1}$ in the form

$$
u^{\prime \prime}(t)=t * A u^{\prime \prime}(t)+g_{1}(t)+1 * g_{2}(t)+t * g_{3}\left[u^{\prime \prime}\right](t)
$$

for $t \in(0, T)$ where

$$
\left.\begin{array}{rl}
g_{1}(t) & =f_{1}(t)+A \varphi \\
g_{2}(t) & =-\sum_{k=1}^{N} m_{k}(t)\left(\widetilde{B}_{k} u\right)(0)+f_{2}(t)+A \psi \\
g_{3}\left[u^{\prime \prime}\right](t) & =-\sum_{k=1}^{N} m_{k} *\left(\widetilde{B}_{k} u\right)^{\prime \prime}(t)-\sum_{k=1}^{N} m_{k}(t)\left(\widetilde{B}_{k} u\right)^{\prime}(0)+f_{3}(t)
\end{array}\right\}
$$

Owing to the assumptions of Lemma 5.1, notation (3.4) and the differentiability of $m_{k}$, we deduce

$$
\begin{aligned}
g_{1} & \in W^{1,1}((0, T) ; Y) \\
g_{2} & \in W^{1,1}\left((0, T) ; Y_{1}\right) \\
g_{3}\left[u^{\prime \prime}\right] & \in W^{1,1}((0, T) ; X)
\end{aligned}
$$

for each $u^{\prime \prime} \in C([0, T] ; Y)$. Now Lemma 4.2/(i) - (ii) imply that equation (5.7) for $u^{\prime \prime} \in C([0, T] ; Y)$ is equivalent to

$$
u^{\prime \prime}(t)=\mathcal{C}(t) g_{1}(0)+\mathcal{C} *\left[g_{1}^{\prime}(t)+\left(1 * g_{2}\right)^{\prime}(t)+\left(t * g_{3}\left[u^{\prime \prime}\right]\right)^{\prime}(t)\right] .
$$

Inserting in this relation $g_{1}, g_{2}, g_{3}$ defined by formulae (5.8), we obtain $(5.3)_{1}$ with (5.4). This shows the equivalence of $(4.1)_{1}$ and $(5.3)_{1}$

\section{Existence, uniqueness and stability}

Let the symbol $P^{k_{1}, \ldots, k_{n}}\left(x_{1}, \ldots, x_{n}\right)$ stand for an arbitrary polynomial of $n$ variables $x_{1}, \ldots, x_{n}$ which has the form

$$
\sum_{i_{1}=0}^{k_{1}} \cdots \sum_{i_{n}=0}^{k_{n}} C_{i_{1}, \ldots, i_{n}} x_{1}^{i_{1}} \cdots x_{n}^{i_{n}}
$$

where $C_{i_{1}, \ldots, i_{n}}$ are non-negative coefficients. 
Theorem 6.1. Let the assumptions of Lemma 5.1 be satisfied for some $N \in \mathbb{N} \cup\{+\infty\}, T \in(0,+\infty)$ and the set of data $d=(\varphi, \psi, f, w, \chi, h)$. Moreover, let

$$
\kappa[d]^{2}=\inf _{\xi \in \mathbb{R}^{N} \backslash\{0\}}\|\xi\|_{\mathbb{R}^{N}}^{-2} \sum_{i, k=1}^{N}\left(\Phi_{i k}[\varphi]+\chi_{i k}(0)\right) \xi_{i} \xi_{k}>0 .
$$

Then identification problem (3.3) has a unique solution $(m, u) \in \mathcal{S}^{N}$. Moreover, the solutions $S^{1}=\left(m^{1}, u^{1}\right)$ and $S^{2}=\left(m^{2}, u^{2}\right)$, corresponding to two sets of data

$$
\begin{aligned}
& d^{1}=\left(\varphi^{1}, \psi^{1}, f^{1}, w^{1}, \chi^{1}, h^{1}\right) \\
& d^{2}=\left(\varphi^{2}, \psi^{2}, f^{2}, w^{2}, \chi^{2}, h^{2}\right)
\end{aligned}
$$

respectively satisfy the stability estimate

$$
\left\|S^{1}-S^{2}\right\|_{N} \leq K\left(\omega_{N}, \kappa\left[d^{1}\right]^{-1}, \kappa\left[d^{2}\right]^{-1},\left|d^{1}\right|_{N},\left|d^{2}\right|_{N}\right)\left|d^{1}-d^{2}\right|_{N}
$$

where

$$
\|S\|_{N}=\|m\|_{H^{1}\left((0, T) ; \mathbb{R}^{N}\right)}+\|u\|_{C^{2}([0, T] ; Y)}
$$

denotes the norm in $\mathcal{S}^{N}, K$ is a function of the form $K=\exp \left(P^{6,3,3,2,2}\right)$ depending also on $T$, the seminorm $|\cdot|_{N}$ and $\omega_{N}$ being given by

$$
\begin{aligned}
|d|_{N}= & \|\varphi\|_{Y}+\|\psi\|_{Y}+\left\|f_{1}+A \varphi\right\|_{W^{1,1}((0, T) ; Y)} \\
& +\left\|f_{2}+A \psi\right\|_{W^{1,1}\left((0, T) ; Y_{1}\right)}+\left\|f_{3}\right\|_{W^{1,1}((0, T) ; X)} \\
& +\left\|w^{\prime}\right\|_{C^{1}\left([0, T] ; X^{N}\right)}+\|B \varphi+w(0)\|_{\left(Y_{1}\right)^{N}} \\
& +\left\|\chi^{\prime}\right\|_{C^{1}\left([0, T] ; \mathcal{L}\left(\mathbb{R}^{N}, \mathbb{R}^{N}\right)\right)}+\left\|h^{\prime}\right\|_{H^{1}\left((0, T) ; \mathbb{R}^{N}\right)}
\end{aligned}
$$

and

$$
\omega_{N}=\|B\|_{(\mathcal{L}(Y, X))^{N}}+\|\Psi\|_{\left(Y^{\star}\right)^{N}}+\left\|\left(\left\|\Phi_{i k}\right\|_{Y^{\star}}\right)_{i, k=1, \ldots, N}\right\|_{\mathcal{L}\left(\mathbb{R}^{N}, \mathbb{R}^{N}\right)} .
$$

Remark 6.1. If the set of data $d=(\varphi, \psi, f, w, \chi, h)$ satisfies assumptions in Theorem 6.1 for some $N \in \mathbb{N} \cup\{+\infty\}$ and any $T \in(0,+\infty)$, then using the standard continuation procedure, we can show that identification problem (3.3), reformulated for $t \in(0,+\infty)$, admits a unique solution $(m, u)$ in the space $H_{l o c}^{1}\left((0,+\infty) ; \mathbb{R}^{N}\right) \times \bigcup_{T>0} C^{2}([0, T] ; Y)$.

Proof of Theorem 6.1. Note first that, due to inequality (6.1) and definition (3.4), relations (5.3) $)_{4}$ determine uniquely the initial values of $m_{k}$. Let us use the abbreviation $m_{k}^{0}=m_{k}(0)$ for these values. Since $u(0)$ and $u^{\prime}(0)$ are also fixed, in order to prove existence and uniqueness in the statement of 
the theorem, we have to show existence and uniqueness in $L^{2}\left((0, T) ; \mathbb{R}^{N}\right) \times$ $C([0, T] ; Y)$ of the solution $\left(m^{\prime}, u^{\prime \prime}\right)$ to subsystem $(5.3)_{1},(5.3)_{3}$.

We begin by proving some auxiliary estimates for $m_{k}^{0}$. By virtue of $(5.3)_{4}$, relation (6.1) and definitions (6.4) - (6.5) we have

$$
\begin{aligned}
\left\|m^{0}[d]\right\|_{\mathbb{R}^{N}} & \leq \kappa[d]^{-1}\left(\|\Psi\|_{\left(Y^{\star}\right)^{N}}\|\psi\|_{Y}+\left\|h^{\prime}(0)\right\|_{\mathbb{R}^{N}}\right) \\
& \leq P^{1,1,1}\left(\omega_{N}, \kappa[d]^{-1},|d|_{N}\right) .
\end{aligned}
$$

For the difference $m^{0}\left[d^{1}\right]-m^{0}\left[d^{2}\right]$ from $(5.3)_{4}$ we deduce the system

$$
\begin{aligned}
& \sum_{k=1}^{N}\left(m_{k}^{0}\left[d^{1}\right]-m_{k}^{0}\left[d^{2}\right]\right)\left(\Phi_{i k}\left[\varphi^{1}\right]+\chi_{i k}^{1}(0)\right) \\
& \quad=\sum_{k=1}^{N} m_{k}^{0}\left[d^{2}\right]\left(\Phi_{i k}\left[\varphi^{2}-\varphi^{1}\right]+\chi_{i k}^{2}(0)-\chi_{i k}^{1}(0)\right)+\Psi_{i}\left[\psi^{1}-\psi^{2}\right]+D_{t}\left(h_{i}^{2}-h_{i}^{1}\right)(0)
\end{aligned}
$$

Reasoning as above, from (6.1) and (6.6) we easily get the estimate

$$
\left\|m^{0}\left[d^{1}\right]-m^{0}\left[d^{2}\right]\right\|_{\mathbb{R}^{N}} \leq P^{2,1,1,1}\left(\omega_{N}, \kappa\left[d^{1}\right]^{-1}, \kappa\left[d^{2}\right]^{-1},\left|d^{2}\right|_{N}\right)\left|d^{1}-d^{2}\right|_{N} .
$$

Let us now come back to system $(5.3)_{1},(5.3)_{3}$. First, we denote the vector $\left(u^{\prime \prime}, m^{\prime}\right)$ by $U$, where $U_{0}=u^{\prime \prime}$ and $U_{k}=m_{k}^{\prime}(k=1, \ldots, N)$. Then we rewrite system $(5.3)_{1},(5.3)_{3}$ as a fixed-point operator equation

$$
U=F U, \quad U \in \mathcal{U}:=C([0, T] ; Y) \times L^{2}\left((0, T) ; \mathbb{R}^{N}\right)
$$

where, in view of (3.4), the components of $F$ are given by the formulae

$$
\begin{aligned}
(F U)_{0}(t)= & -\sum_{k=1}^{N}\left[m_{k}^{0}+U_{k} *\right]\left[t * \mathcal{C} *\left(B_{k} U_{0}(t)+w_{k}^{\prime \prime}(t)\right)\right. \\
& \left.+t * \mathcal{C}(t)\left(B_{k} \psi+w_{k}^{\prime}(0)\right)+1 * \mathcal{C}(t)\left(B_{k} \varphi+w_{k}(0)\right)\right]+\alpha(t)
\end{aligned}
$$

and

$$
\sum_{k=1}^{N}\left(\Phi_{i k}[\varphi]+\chi_{i k}(0)\right)(F U)_{k}(t)=(G U)_{i}(t)
$$

for $i=1, \ldots, N$ with

$$
\begin{aligned}
(G U)_{i}(t)= & -\sum_{k=1}^{N}\left\{U_{k} * 1 *\left(\Phi_{i k}\left[U_{0}(t)\right]+\chi_{i k}^{\prime \prime}(t)\right)+U_{k} *\left(\Phi_{i k}[\psi]+\chi_{i k}^{\prime}(0)\right)\right. \\
& \left.+m_{k}^{0} *\left(\Phi_{i k}\left[U_{0}(t)\right]+\chi_{i k}^{\prime \prime}(t)\right)\right\}+\Psi_{i}\left[(F U)_{0}(t)\right] \\
& -h_{i}^{\prime \prime}(t)-\sum_{k=1}^{N} m_{k}^{0}\left(\Phi_{i k}[\psi]+\chi_{i k}^{\prime}(0)\right)
\end{aligned}
$$


Our aim is to apply the contraction mapping principle to equation (6.8). To this end we have to introduce proper norms and derive some estimates for the components of $F$. Therefore, let us equip the space $\mathcal{U}$ with the scale of weighted norms depending on the real parameter $\gamma \geq 0$

$$
\|U\|_{\gamma}=\left\|U_{0}\right\|_{\infty, \gamma}+\left(\sum_{k=1}^{N}\left\|U_{k}\right\|_{2, \gamma}^{2}\right)^{1 / 2}
$$

where

$$
\left.\begin{array}{rl}
\left\|U_{0}\right\|_{\infty, \gamma} & =\left\|e^{-\gamma t} U_{0}\right\|_{C([0, T] ; Y)} \\
\left\|U_{k}\right\|_{2, \gamma} & =\left\|e^{-\gamma t} U_{k}\right\|_{L^{2}(0, T)}
\end{array}\right\} .
$$

Let us now formulate a boundedness lemma, whose proof we postpone to the last section of the paper.

Lemma 6.1. Under the assumptions in Theorem 6.1 the estimates

$$
\begin{aligned}
\|(F[d] U)\|_{\gamma} \leq & K_{1}\left(\omega_{N}, \kappa[d]^{-1},|d|_{N}\right)\left(1+\gamma^{-1 / 2}\|U\|_{\gamma}\right) \\
& +K_{2}\left(\omega_{N}, \kappa[d]^{-1}\right) \gamma^{-1}\|U\|_{\gamma}^{2}
\end{aligned}
$$

and

$$
\begin{aligned}
&\left\|F\left[d^{1}\right] U^{1}-F\left[d^{2}\right] U^{2}\right\|_{\gamma} \\
& \leq K_{3}\left(\omega_{N}, \kappa\left[d^{1}\right]^{-1}, \kappa\left[d^{2}\right]^{-1},\left|d^{1}\right|_{N},\left|d^{2}\right|_{N},\left\|U^{1}\right\|_{\gamma},\left\|U^{2}\right\|_{\gamma}\right)\left|d^{1}-d^{2}\right|_{N} \\
&+\left\{K_{4}\left(\omega_{N}, \kappa\left[d^{1}\right]^{-1}, \kappa\left[d^{2}\right]^{-1},\left|d^{1}\right|_{N},\left|d^{2}\right|_{N}\right)\right. \\
&\left.+K_{5}\left(\omega_{N}, \kappa\left[d^{1}\right]^{-1}\right)\left(\left\|U^{1}\right\|_{\gamma}+\left\|U^{2}\right\|_{\gamma}\right)\right\} \gamma^{-1 / 2}\left\|U^{1}-U^{2}\right\|_{\gamma}
\end{aligned}
$$

are valid where $K_{1}=P^{3,2,2}, K_{2}=P^{2,1}, K_{3}=P^{6,2,2,1,2,1,2}, K_{4}=P^{4,1,1,1,1}$ and $K_{5}=P^{3,1}$.

In order to prove the existence and uniqueness of the solution of (6.8) we need the closed balls

$$
D_{\gamma}(r)=\left\{U \in \mathcal{U}:\|U\|_{\gamma} \leq r\right\}
$$

Then for any $U \in D_{\gamma}(r)$ from (6.14) it follows

$$
\|F[d] U\|_{\gamma} \leq K_{1}\left(\omega_{N}, \kappa[d]^{-1},|d|_{N}\right)\left(1+r \gamma^{-1 / 2}\right)+K_{2}\left(\omega_{N}, \kappa[d]^{-1}\right) r^{2} \gamma^{-1} .
$$

This estimate shows that for every $r>K_{1}\left(\omega_{N}, \kappa[d]^{-1},|d|_{N}\right)$ and

$$
\gamma \geq \gamma_{1}\left(\omega_{N}, \kappa[d]^{-1},|d|_{N}, r\right):=\left[\frac{2 K_{2} r}{\sqrt{K_{1}^{2}+4 K_{2}\left(r-K_{1}\right)}-K_{1}}\right]^{2}
$$


the inequality $\|F[d] U\|_{\gamma} \leq r$ holds. Hence

$$
F[d]: D_{\gamma}(r) \rightarrow D_{\gamma}(r)
$$

if

$$
\begin{aligned}
& r>K_{1}\left(\omega_{N}, \kappa[d]^{-1},|d|_{N}\right) \\
& \gamma \geq \gamma_{1}\left(\omega_{N}, \kappa[d]^{-1},|d|_{N}, r\right) .
\end{aligned}
$$

Next let us show that $F[d]$ is a contraction mapping for any fixed data. Suppose that $U^{1}, U^{2} \in D_{\gamma}(r)$. Then from (6.15) we obtain the estimate

$$
\begin{aligned}
& \left\|F[d] U^{1}-F[d] U^{2}\right\|_{\gamma} \\
& \quad \leq\left\{K_{4}\left(\omega_{N}, \kappa[d]^{-1}, \kappa[d]^{-1},|d|_{N},|d|_{N}\right)+2 K_{5}\left(\omega_{N}, \kappa[d]^{-1}\right) r\right\} \gamma^{-\frac{1}{2}}\left\|U^{1}-U^{2}\right\|_{\gamma}
\end{aligned}
$$

Defining

$$
\begin{aligned}
& \gamma_{2}\left(\omega_{N}, \kappa[d]^{-1},|d|_{N}, r\right) \\
& \quad=4\left[K_{4}\left(\omega_{N}, \kappa[d]^{-1}, \kappa[d]^{-1},|d|_{N},|d|_{N}\right)+2 K_{5}\left(\omega_{N}, \kappa[d]^{-1}\right) r\right]^{2}
\end{aligned}
$$

the operator $F$ turns out to be a contraction mapping with respect to the norm $\|\cdot\|_{\gamma}$ if $\gamma \geq \gamma_{2}\left(\omega_{N}, \kappa[d]^{-1},|d|_{N}, r\right)$. This, together with (6.17), proves that operator equation (6.8) has a unique solution in each ball $D_{\gamma}(r)$ such that

$$
\begin{aligned}
& r>K_{1}\left(\omega_{N}, \kappa[d]^{-1},|d|_{N}\right) \\
& \gamma \geq \gamma_{3}\left(\omega_{N}, \kappa[d]^{-1},|d|_{N}, r\right)
\end{aligned}
$$

where

$$
\begin{aligned}
& \gamma_{3}\left(\omega_{N}, \kappa[d]^{-1},|d|_{N}, r\right) \\
& \quad=\max \left\{\gamma_{1}\left(\omega_{N}, \kappa[d]^{-1},|d|_{N}, r\right), \gamma_{2}\left(\omega_{N}, \kappa[d]^{-1},|d|_{N}, r\right)\right\} .
\end{aligned}
$$

In particular, this proves our existence assertion.

Next let us prove the uniqueness of the solution $U$. To this end we have to show that the solution of $(6.8)$ is unique in the whole space $\mathcal{U}$. Suppose that (6.8) has two solutions $U^{1}$ and $U^{2}$ in $\mathcal{U}$. Let us fix any

$$
r_{1}>\max \left\{\left\|U^{1}\right\|_{0},\left\|U^{2}\right\|_{0}, K_{1}\left(\omega_{N}, \kappa[d]^{-1},|d|_{N}\right)\right\}
$$

Then $\left\|U^{i}\right\|_{\gamma} \leq\left\|U^{i}\right\|_{0} \leq r_{1} \quad(i=1,2)$ for each $\gamma \geq 0$ because of the monotonicity of the norm $\|\cdot\|_{\gamma}$ with respect to $\gamma$. In particular, these inequalities hold for $\gamma \geq \gamma_{3}\left(\omega_{N}, \kappa[d]^{-1},|d|_{N}, r_{1}\right)$, too. This means that $U^{i}$ belongs to the 
ball $D_{\gamma}\left(r_{1}\right)$ for $\gamma \geq \gamma_{3}\left(\omega_{N}, \kappa[d]^{-1},|d|_{N}, r_{1}\right)$ and $i=1,2$. But in these balls the uniqueness has already been shown. Thus, we obtain $U^{1}=U^{2}$.

It remains to prove stability estimate (6.2). Let $U^{1}$ and $U^{2}$ be the solutions of (6.8) corresponding to the data sets $d^{1}$ and $d^{2}$, respectively. By virtue of (6.15) from equation (6.8) we obtain the estimate

$$
\begin{aligned}
\left\|U^{1}-U^{2}\right\|_{\gamma}= & \left\|F\left[d^{1}\right] U^{1}-F\left[d^{2}\right] U^{2}\right\|_{\gamma} \\
\leq & K_{3}\left(\omega_{N}, \kappa\left[d^{1}\right]^{-1}, \kappa\left[d^{2}\right]^{-1},\left|d^{1}\right|_{N},\left|d^{2}\right|_{N},\left\|U^{1}\right\|_{\gamma},\left\|U^{2}\right\|_{\gamma}\right)\left|d^{1}-d^{2}\right|_{N} \\
& +\left\{K_{4}\left(\omega_{N}, \kappa\left[d^{1}\right]^{-1}, \kappa\left[d^{2}\right]^{-1},\left|d^{1}\right|_{N},\left|d^{2}\right|_{N}\right)\right. \\
& \left.+K_{5}\left(\omega_{N}, \kappa\left[d^{1}\right]^{-1}\right)\left(\left\|U^{1}\right\|_{\gamma}+\left\|U^{2}\right\|_{\gamma}\right)\right\} \gamma^{-1 / 2}\left\|U^{1}-U^{2}\right\|_{\gamma}
\end{aligned}
$$

From the previous proof concerning the existence of the solution to (6.8) we know that $U^{i} \in D_{\gamma}(r)$ if

$$
\begin{aligned}
& r>K_{1}\left(\omega_{N}, \kappa\left[d^{i}\right]^{-1}\left|d^{i}\right|_{N}\right) \\
& \gamma \geq \gamma_{3}\left(\omega_{N}, \kappa\left[d^{i}\right]^{-1},\left|d^{i}\right|_{N}, r\right) .
\end{aligned}
$$

Let us now choose the values of $r$ and $\gamma$ according to the formulae

$$
\left.\begin{array}{rl}
\bar{r}= & 2 \max \left\{K_{1}\left(\omega_{N}, \kappa\left[d^{1}\right]^{-1},\left|d^{1}\right|_{N}\right), K_{1}\left(\omega_{N}, \kappa\left[d^{2}\right]^{-1},\left|d^{2}\right|_{N}\right)\right\} \\
\bar{\gamma}= & \max \left\{\gamma_{3}\left(\omega_{N}, \kappa\left[d^{1}\right]^{-1},\left|d^{1}\right|_{N}, \bar{r}\right), \gamma_{3}\left(\omega_{N}, \kappa\left[d^{2}\right]^{-1},\left|d^{2}\right|_{N}, \bar{r}\right),\right. \\
& \left.4\left[K_{4}\left(\omega_{N}, \kappa\left[d^{1}\right]^{-1}, \kappa\left[d^{2}\right]^{-1},\left|d^{1}\right|_{N},\left|d^{2}\right|_{N}\right)+2 K_{5}\left(\omega_{N}, \kappa\left[d^{1}\right]^{-1}\right) \bar{r}\right]^{2}\right\} .
\end{array}\right\}
$$

Hence $U^{i} \in D_{\bar{\gamma}}(\bar{r}) \quad(i=1,2)$, i.e.

$$
\left\|U^{i}\right\|_{\bar{\gamma}} \leq \bar{r} \quad(i=1,2) .
$$

Let us now set $\gamma=\bar{\gamma}$ in (6.20). From (6.21) - (6.22) we deduce that the coefficient of $\left\|U^{1}-U^{2}\right\|_{\gamma}$ on the right-hand side of (6.20) is less than $\frac{1}{2}$. Thus we have

$$
\left\|U^{1}-U^{2}\right\|_{\bar{\gamma}} \leq 2 K_{3}\left(\omega_{N}, \kappa\left[d^{1}\right]^{-1}, \kappa\left[d^{2}\right]^{-1},\left|d^{1}\right|_{N},\left|d^{2}\right|_{N}, \bar{r}, \bar{r}\right)\left|d^{1}-d^{2}\right|_{N} .
$$

Using the obvious relation $\|\cdot\|_{0} \leq e^{\gamma T}\|\cdot\|_{\gamma}$, we obtain

$$
\left\|U^{1}-U^{2}\right\|_{0} \leq 2 e^{\bar{\gamma} T} K_{3}\left(\omega_{N}, \kappa\left[d^{1}\right]^{-1}, \kappa\left[d^{2}\right]^{-1},\left|d^{1}\right|_{N},\left|d^{2}\right|_{N}, \bar{r}, \bar{r}\right)\left|d^{1}-d^{2}\right|_{N} .
$$


Elementary computations involving the definitions of $\bar{\gamma}$ and $\bar{r}$, which in turn use the definitions of $\gamma_{1}, \ldots, \gamma_{3}$, and the degrees of the polynomials $K_{1}, K_{2}$ and $K_{4}, K_{5}$ (cf. Lemma 6.1) show that

$$
\left.\begin{array}{l}
\bar{\gamma} \leq P^{6,3,3,2,2}\left(\omega_{N}, \kappa\left[d^{1}\right]^{-1}, \kappa\left[d^{2}\right]^{-1},\left|d^{1}\right|_{N},\left|d^{2}\right|_{N}\right) \\
\bar{r}=P^{3,2,2,2,2}\left(\omega_{N}, \kappa\left[d^{1}\right]^{-1}, \kappa\left[d^{2}\right]^{-1},\left|d^{1}\right|_{N},\left|d^{2}\right|_{N}\right)
\end{array}\right\} .
$$

We recall that

$$
\left\|U^{1}-U^{2}\right\|_{0}=\left\|D_{t}\left(m^{1}-m^{2}\right)\right\|_{L^{2}\left((0, T) ; \mathbb{R}^{N}\right)}+\left\|D_{t}^{2}\left(u^{1}-u^{2}\right)\right\|_{C([0, T] ; Y)} .
$$

Consequently, from (6.25), (6.23), (6.7) and the estimates

$$
\begin{aligned}
\left\|u^{1}(0)-u^{2}(0)\right\|_{Y} & =\left\|\varphi^{1}-\varphi^{2}\right\|_{Y} \leq\left|d^{1}-d^{2}\right|_{N} \\
\left\|D_{t} u^{1}(0)-D_{t} u^{2}(0)\right\|_{Y} & =\left\|\psi^{1}-\psi^{2}\right\|_{Y} \leq\left|d^{1}-d^{2}\right|_{N}
\end{aligned}
$$

for the initial values of $u^{1}-u^{2}$, we obtain the desired estimate (6.2). The assertion $K=\exp \left(P^{6,3,3,2,2}\right)$ follows from (6.24) and the order of $K_{3}$ in Lemma 6.1

\section{Approximation results}

In this section we study whether the solutions of the identification problem corresponding to finite $N$ 's, i.e. to degenerate kernels, approximate the solution corresponding to $N=+\infty$, i.e. to a non-degenerate kernel.

Suppose that we are given a sequence of data

$$
d^{n}=\left(\varphi^{n}, \psi^{n}, f^{n}, w^{n}, \chi^{n}, h^{n}\right) \quad \text { with }\left\{\begin{array}{l}
w^{n}=\left(w_{k}^{n}\right)_{k=1, \ldots, n} \\
\chi^{n}=\left(\chi_{i k}^{n}\right)_{i, k=1, \ldots, n} \\
h^{n}=\left(h_{i}^{n}\right)_{i=1, \ldots, n}
\end{array} \quad(n \in \mathbb{N})\right.
$$

and a set of data

$$
d^{\infty}=\left(\varphi^{\infty}, \psi^{\infty}, f^{\infty}, w^{\infty}, \chi^{\infty}, h^{\infty}\right) \quad \text { with }\left\{\begin{array}{l}
w^{\infty}=\left(w_{k}^{\infty}\right)_{k=1, \ldots, \infty} \\
\chi^{\infty}=\left(\chi_{i k}^{\infty}\right)_{i, k=1, \ldots, \infty} \\
h^{\infty}=\left(h_{i}^{\infty}\right)_{i=1, \ldots, \infty}
\end{array}\right.
$$

By

$$
\begin{aligned}
S^{n} & =\left(m^{n}, u^{n}\right) & \text { with } & m^{n}=\left(m_{k}^{n}\right)_{k=1, \ldots n} \\
S^{\infty} & =\left(m^{\infty}, u^{\infty}\right) & \text { with } & m^{\infty}=\left(m_{k}^{\infty}\right)_{k=1, \ldots \infty}
\end{aligned}
$$


we denote the solutions to identification problem (3.3) corresponding to the cases $N=n, d=d^{n}$ and $N=+\infty, d=d^{\infty}$, respectively. In the latter case we simply assume that a solution $S^{\infty}$ does exist. Let us then define a projector $Q^{n}$ mapping $S^{\infty}$ into a finite-dimensional space according to the rule

$$
Q^{n} S^{\infty}=\left(Q_{1}^{n} m^{\infty}, u^{\infty}\right) \quad \text { where } Q_{1}^{n} m^{\infty}=\left(m_{k}^{\infty}\right)_{k=1, \ldots, n}
$$

We now ask the following question:

What conditions must satisfy $d^{n}, d^{\infty}$ and, possibly, $S^{\infty}$ to guarantee the convergence $\left\|S^{n}-Q^{n} S^{\infty}\right\|_{n} \rightarrow 0$ as $n \rightarrow+\infty$ ?

In order to answer this question, let us first perform some formal computations. Let us rewrite relations $(3.3)_{1-2}$ and the first $n$ equations of system $(3.3)_{3}$ occurring in the identification problem for $S^{\infty}$ in the form

$$
\left.\begin{array}{l}
D_{t}^{2} u^{\infty}(t)=A u^{\infty}(t)-\sum_{k=1}^{n} m_{k}^{\infty} *\left(B_{k} u^{\infty}(t)+w_{k}^{\infty}(t)\right)+\widetilde{f}^{\infty, n}(t) \\
u^{\infty}(0)=\varphi^{\infty}, \quad D_{t} u^{\infty}(0)=\psi^{\infty} \\
\Psi_{i}\left[u^{\infty}(t)\right]-\sum_{k=1}^{n} m_{k}^{\infty} *\left(\Phi_{i k}\left[u^{\infty}(t)\right]+\chi_{i k}^{\infty}(t)\right)=\widetilde{h}_{i}^{\infty, n}(t)
\end{array}\right\}
$$

for $t \in(0, T)$ and $i=1, \ldots, n$ where

$$
\left.\begin{array}{l}
\widetilde{f}^{\infty, n}(t)=f^{\infty}(t)-\sum_{k=n+1}^{+\infty} m_{k}^{\infty} *\left(B_{k} u^{\infty}(t)+w_{k}^{\infty}(t)\right) \\
\widetilde{h}_{i}^{\infty, n}(t)=h_{i}^{\infty}(t)+\sum_{k=n+1}^{+\infty} m_{k}^{\infty} *\left(\Phi_{i k}\left[u^{\infty}(t)\right]+\chi_{i k}^{\infty}(t)\right)
\end{array}\right\} .
$$

The function $Q^{n} S^{\infty}$ solves identification problem (7.1) or, in other words, is the solution to identification problem (3.3) with $N=n$ and data $d=\widetilde{d}^{n}$ where

$$
\widetilde{d}^{n}=\left(\varphi^{\infty}, \psi^{\infty}, \widetilde{f}^{\infty, n}, P_{4}^{n} w^{\infty}, P_{5}^{n} \chi^{\infty}, \widetilde{h}^{\infty, n}\right) \quad \text { with }\left\{\begin{array}{c}
P_{4}^{n} w^{\infty}=\left(w_{k}^{\infty}\right)_{k=1, \ldots, n} \\
P_{5}^{n} \chi^{\infty}=\left(\chi_{i k}^{\infty}\right)_{i, k=1, \ldots, n}
\end{array}\right.
$$

Applying stability estimate (6.2) in Theorem 6.1, we get the relation

$$
\left\|S^{n}-Q^{n} S^{\infty}\right\|_{n} \leq K\left(\omega_{n}, \kappa\left[d^{n}\right]^{-1}, \kappa\left[\widetilde{d}^{n}\right]^{-1},\left|d^{n}\right|_{n},\left|\widetilde{d}^{n}\right|_{n}\right)\left|d^{n}-\widetilde{d}^{n}\right|_{n}
$$


Here $\omega_{n}$ is given by $(6.5)$ and $\kappa\left[d^{n}\right], \kappa\left[\widetilde{d}^{n}\right]$ are defined by

$$
\left.\begin{array}{l}
\kappa\left[d^{n}\right]^{2}=\inf _{\xi \in \mathbb{R}^{n} \backslash\{0\}}\|\xi\|_{\mathbb{R}^{n}}^{-2} \sum_{i, k=1}^{n}\left(\Phi_{i k}\left[\varphi^{n}\right]+\chi_{i k}^{n}(0)\right) \xi_{i} \xi_{k}>0 \\
\kappa\left[\widetilde{d}^{n}\right]^{2}=\inf _{\xi \in \mathbb{R}^{n} \backslash\{0\}}\|\xi\|_{\mathbb{R}^{n}}^{-2} \sum_{i, k=1}^{n}\left(\Phi_{i k}\left[\varphi^{\infty}\right]+\chi_{i k}^{\infty}(0)\right) \xi_{i} \xi_{k}>0
\end{array}\right\} .
$$

In order to obtain the desired relation $\left\|S^{n}-Q^{n} S^{\infty}\right\|_{n} \rightarrow 0 \quad(n \rightarrow+\infty)$ it suffices to assume that $\left|d^{n}-\widetilde{d}^{n}\right|_{n} \rightarrow 0(n \rightarrow+\infty)$ so fast as to compensate the (possible) increase of the factor $K\left(\omega_{n}, \kappa\left[d^{n}\right]^{-1}, \kappa\left[\widetilde{d}^{n}\right]^{-1},\left|d^{n}\right|_{n},\left|\widetilde{d}^{n}\right|_{n}\right)$ in (7.4).

Summing up, we can formulate the following

Theorem 7.1. Let the operators $B=\left(B_{k}\right)_{k=1, \ldots, \infty}, \Psi=\left(\Psi_{i}\right)_{i=1, \ldots, \infty}$ and $\Phi=\left(\Phi_{i k}\right)_{i, k=1, \ldots, \infty}$ satisfy (3.1) - (3.2) with $N=+\infty$. Assume that each $d^{n} \quad(n \in \mathbb{N})$ fulfills the conditions in Lemma 5.1 with $N=n$ and suppose that when $N=+\infty$, problem (3.3) with data $d^{\infty}$ admits a solution $S^{\infty}$ such that $Q^{n} S^{\infty} \in \mathcal{S}^{n}$ for any $n \in \mathbb{N}$. Further, assume that the data vector $\widetilde{d}^{n}(n \in \mathbb{N})$, which is defined by means of the components of $S^{\infty}$ and $d^{\infty}$, satisfies the assumptions in Lemma 5.1 with $N=n$. Moreover, let (7.5) hold. If

$$
K\left(\omega_{n}, \kappa\left[d^{n}\right]^{-1}, \kappa\left[\widetilde{d}^{n}\right]^{-1},\left|d^{n}\right|_{n},\left|\widetilde{d}^{n}\right|_{n}\right)\left|d^{n}-\widetilde{d}^{n}\right|_{n} \rightarrow 0 \quad \text { as } n \rightarrow+\infty
$$

then $\left\|S^{n}-Q^{n} S^{\infty}\right\|_{n} \rightarrow 0$ as $n \rightarrow+\infty$.

Theorem 7.1 yields the following corollary:

Corollary 7.1. If $B, \Psi, \Phi$ and $d^{n}$ satisfy the assumptions in Theorem 7.1, then problem (3.3) with $N=+\infty$ and data $d^{\infty}$ admits at most one solution $S^{\infty}$ such that $Q^{n} S^{\infty} \in \mathcal{S}^{n}$ for any $n \in \mathbb{N}$, whenever the $\widetilde{d}^{n}$ 's satisfy the assumptions in Lemma 5.1 with $N=n \quad(n \in \mathbb{N})$ and inequality $(7.5)_{2}$ as well as convergence relation (7.6).

Proof. Suppose that problem (3.3) with $N=+\infty$ and $d=d^{\infty}$ has two solutions $S_{1}^{\infty}$ and $S_{2}^{\infty}$ fulfilling the properties listed in Corollary 7.1. Then Theorem 7.1 implies that the solution $S^{n}$ of problem (3.3) with $N=n$ and $d=d^{n}$, which in turn exists and is unique by Theorem 6.1 , satisfies the convergence relations

$$
\left.\begin{array}{l}
\left\|S^{n}-Q^{n} S_{1}^{\infty}\right\|_{n} \\
\left\|S^{n}-Q^{n} S_{2}^{\infty}\right\|_{n}
\end{array}\right\} \rightarrow 0 \text { as } n \rightarrow+\infty
$$


In particular, $\left\|Q^{n}\left(S_{1}^{\infty}-S_{2}^{\infty}\right)\right\|_{n} \rightarrow 0$ as $n \rightarrow+\infty$. In other words, we have proved that

$$
\begin{aligned}
& \left(\sum_{k=1}^{+\infty}\left\|m_{k}^{1, \infty}-m_{k}^{2, \infty}\right\|_{H^{1}\left((0, T) ; \mathbb{R}^{N}\right)}^{2}\right)^{\frac{1}{2}}+\left\|u^{1, \infty}-u^{2, \infty}\right\|_{C^{2}([0, T] ; Y)} \\
& \quad=\lim _{n \rightarrow+\infty}\left[\left(\sum_{k=1}^{n}\left\|m_{k}^{1, \infty}-m_{k}^{2, \infty}\right\|_{H^{1}\left((0, T) ; \mathbb{R}^{N}\right)}^{2}\right)^{\frac{1}{2}}+\left\|u^{1, \infty}-u^{2, \infty}\right\|_{C^{2}([0, T] ; Y)}\right] \\
& \quad=\lim _{n \rightarrow+\infty}\left\|Q^{n}\left(S_{1}^{\infty}-S_{2}^{\infty}\right)\right\|_{n} \\
& \quad=0 .
\end{aligned}
$$

This implies $m_{k}^{1, \infty}=m_{k}^{2, \infty} \quad(k \in \mathbb{N})$ and $u^{1, \infty}=u^{2, \infty}$, i.e. $S_{1}^{\infty}=S_{2}^{\infty}$

We now note that relation (7.6) occurs when the factor

$$
K\left(\omega_{n}, \kappa\left[d^{n}\right]^{-1}, \kappa\left[\widetilde{d}^{n}\right]^{-1},\left|d^{n}\right|_{n},\left|\widetilde{d}^{n}\right|_{n}\right)
$$

is bounded as $n \rightarrow+\infty$. This is related to the case when $d^{\infty}$ and the operator vectors $B, \Psi, \Phi$ fulfill the conditions of Theorem 6.1 with $N=+\infty$. In order to treat this case separately, let us first introduce the projector $P_{n}$ which maps $d^{\infty}$ into a finite-dimensional space defined by

$$
P_{n} d^{\infty}=\left(\varphi^{\infty}, \psi^{\infty}, f^{\infty}, P_{4}^{n} w^{\infty}, P_{5}^{n} \chi^{\infty}, P_{6}^{n} h^{\infty}\right)
$$

where $P_{6}^{n} h^{\infty}=\left(h_{i}^{\infty}\right)_{i=1, \ldots, n}$ and $P_{4}^{n}, P_{5}^{n}$ are defined by (7.3). Then we can prove the following

Theorem 7.2. Let the operators $B, \Psi, \Phi$ satisfy (3.1) - (3.2) with $N=$ $+\infty$ and (5.2). Assume that $d^{n}$ and $d^{\infty}$ fulfill the conditions in Lemma 5.1 with $N=n(n \in \mathbb{N})$ and $N=+\infty$, respectively. Moreover, let the condition

$$
\kappa^{2}=\inf _{n \in \mathbb{N}} \inf _{\xi \in \mathbb{R}^{n} \backslash\{0\}}\|\xi\|_{\mathbb{R}^{n}}^{-2} \sum_{i, k=1}^{n}\left(\Phi_{i k}\left[\varphi^{n}\right]+\chi_{i k}^{n}(0)\right) \xi_{i} \xi_{k}>0
$$

hold. If

$$
\left|d^{n}-P^{n} d^{\infty}\right|_{n} \rightarrow 0 \quad \text { as } n \rightarrow+\infty,
$$

then $\left\|S^{n}-Q^{n} S^{\infty}\right\|_{n} \rightarrow 0$ as $n \rightarrow+\infty$.

Remark 7.1. It is easy to check, using convergence assumption (7.9), that condition (7.8) yields the analogous condition

$$
\kappa_{1}^{2}=\inf _{\xi \in \mathbb{R}^{\infty} \backslash\{0\}}\|\xi\|_{\mathbb{R}^{\infty}}^{-2} \sum_{i, k=1}^{+\infty}\left(\Phi_{i k}\left[\varphi^{\infty}\right]+\chi_{i k}^{\infty}(0)\right) \xi_{i} \xi_{k}>0 .
$$


Proof of Theorem 7.2. Theorem 6.1 implies the existence of solutions $S^{n}=\left(m^{n}, u^{n}\right) \in \mathcal{S}^{n}$ and $S^{\infty}=\left(m^{\infty}, u^{\infty}\right) \in \mathcal{S}^{\infty}$ to identification problems (3.3) corresponding to $N=n, d=d^{n}$ and $N=\infty, d=d^{\infty}$, respectively. From (7.2) - (7.3) and (7.7) we obtain

$$
\widetilde{d}^{n}=P^{n} d^{\infty}+\delta^{n} \quad \text { where } \delta^{n}=\left(0,0, \widehat{f}^{\infty, n}, 0,0, \widehat{h}^{\infty, n}\right)
$$

and

$$
\begin{aligned}
\widehat{f}^{\infty, n}(t) & =-\sum_{k=n+1}^{+\infty} m_{k}^{\infty} *\left(B_{k} u^{\infty}(t)+w_{k}^{\infty}(t)\right) \\
\widehat{h}^{\infty, n} & =\left(\widehat{h}_{i}^{\infty, n}\right)_{i=1, \ldots, n}, \quad \widehat{h}_{i}^{\infty, n}(t)=\sum_{k=n+1}^{+\infty} m_{k}^{\infty} *\left(\Phi_{i k}\left[u^{\infty}(t)\right]+\chi_{i k}^{\infty}(t)\right) .
\end{aligned}
$$

Let us transform the addenda of the series appearing in the formula of $\widehat{f}^{\infty, n}$ in the following way:

$$
\begin{aligned}
m_{k}^{\infty} * & \left(B_{k} u^{\infty}(t)+w_{k}^{\infty}(t)\right) \\
= & m_{k}^{\infty} *\left[1 *\left(B_{k} D_{t} u^{\infty}(t)+D_{t} w_{k}^{\infty}(t)\right)+B_{k} \varphi^{\infty}+w_{k}^{\infty}(0)\right] \\
= & \left(m_{k}^{\infty}(0)+1 * D_{t} m_{k}^{\infty}\right) * 1 *\left(B_{k} D_{t} u^{\infty}(t)+D_{t} w_{k}^{\infty}(t)\right) \\
& +1 *\left[m_{k}^{\infty}(t)\left(B_{k} \varphi^{\infty}+w_{k}^{\infty}(0)\right)\right] .
\end{aligned}
$$

We can now rewrite $\widehat{f}^{\infty, n}$ in the form

$$
\widehat{f}^{\infty, n}=1 * \widehat{f}_{2}^{\infty, n}+t * \widehat{f}_{3}^{\infty, n}
$$

where

$$
\begin{aligned}
\widehat{f}_{2}^{\infty, n}(t)= & -\sum_{k=n+1}^{+\infty} m_{k}^{\infty}(t)\left(B_{k} \varphi^{\infty}+w_{k}^{\infty}(0)\right) \\
\widehat{f}_{3}^{\infty, n}(t)= & -\sum_{k=n+1}^{+\infty}\left[m_{k}^{\infty}(0)\left(B_{k} D_{t} u^{\infty}(t)+D_{t} w_{k}^{\infty}(t)\right)\right. \\
& \left.+D_{t} m_{k}^{\infty} *\left(B_{k} D_{t} u^{\infty}(t)+D_{t} w_{k}^{\infty}(t)\right)\right] .
\end{aligned}
$$

Taking advantage of the assumptions made on $d^{\infty}$, of conditions (5.2) and the membership $\left(m^{\infty}, u^{\infty}\right) \in \mathcal{S}^{\infty}$, we can immediately check that

$$
\begin{aligned}
& \widehat{f}_{2}^{\infty, n} \in W^{1,1}\left((0, T) ; Y_{1}\right) \\
& \widehat{f}_{3}^{\infty, n} \in W^{1,1}((0, T) ; X) \\
& \widehat{h}^{\infty, n} \in H^{2}\left((0, T) ; \mathbb{R}^{n}\right)
\end{aligned}
$$


and

$$
\begin{aligned}
\left|\delta^{n}\right|_{n}= & \left\|\widehat{f}_{2}^{\infty, n}\right\|_{W^{1,1}\left((0, T) ; Y_{1}\right)} \\
& +\left\|\widehat{f}_{3}^{\infty, n}\right\|_{W^{1,1}((0, T) ; X)} \\
& +\left\|D_{t} \widehat{h}^{\infty, n}\right\|_{H^{1}\left((0, T) ; \mathbb{R}^{n}\right)} \\
& \rightarrow 0 \text { as } n \rightarrow+\infty
\end{aligned}
$$

Since $P^{n} d^{\infty}$ satisfies the assumptions in Theorem 6.1 with $N=n, \widetilde{d}^{n}$ satisfies the same assumptions. Therefore, problem (3.3) with data $\widetilde{d}^{n}$ can be solved when $N=n$, and the solution coincides with $Q^{n} S^{\infty}$. From Theorem 6.1 we have the stability estimate

$$
\left\|S^{n}-Q^{n} S^{\infty}\right\|_{n} \leq K\left(\omega_{n}, \kappa^{-1}, \kappa_{1}^{-1},\left|d^{n}\right|_{n},\left|\widetilde{d}^{n}\right|_{n}\right)\left|d^{n}-\widetilde{d}^{n}\right|_{n}
$$

where $\omega_{n}$ is given by (6.5) and where $\kappa$ and $\kappa_{1}$ are the constants defined in (7.8) and (7.10), respectively. From (7.9) and (7.11) - (7.12) we deduce

$$
\left|d^{n}-\widetilde{d}^{n}\right|_{n} \leq\left|d^{n}-P^{n} d^{\infty}\right|_{n}+\left|\delta^{n}\right|_{n} \rightarrow 0 \quad \text { as } n \rightarrow+\infty
$$

Besides, $\omega_{n} \leq \omega_{\infty}$, due to $(5.2)$, and

$$
\left|d^{n}\right|_{n} \leq\left|d^{n}-P^{n} d^{\infty}\right|_{n}+\left|P^{n} d^{\infty}\right|_{n} \leq\left|d^{n}-P^{n} d^{\infty}\right|_{n}+\left|d^{\infty}\right|_{\infty} \leq \mathrm{const}
$$

as $n \rightarrow+\infty$, which in turn implies $\left|\widetilde{d}^{n}\right|_{n} \leq\left|\widetilde{d}^{n}-d^{n}\right|_{n}+\left|d^{n}\right|_{n} \leq$ const as $n \rightarrow+\infty$ by (7.14). Now from (7.13) we obtain $\left\|S^{n}-Q^{n} S^{\infty}\right\|_{n} \rightarrow 0$ as $n \rightarrow+\infty$

\section{Application to a viscoelastic problem}

We are now going to apply the results of Sections 3 - 7 to viscoelastic identification problem (2.3) - (2.5), (2.8) posed in Section 2. To this end we first introduce the functional spaces

$$
\begin{aligned}
& X=L^{2}(\Omega) \\
& Y=H^{2}(\Omega) \cap H_{0}^{1}(\Omega)
\end{aligned}
$$

and the linear differential operators

$$
\begin{aligned}
A v & =\operatorname{div}(\lambda \nabla v) \\
B_{k} v & =\operatorname{div}\left(\mu_{k} \nabla v\right) \quad(k=1, \ldots, N) .
\end{aligned}
$$


Assume that

$$
\begin{aligned}
\lambda \in W^{1, r}(\Omega), \lambda(x) \geq \lambda_{0}>0 \text { for a.e. } x \in \Omega \\
\mu_{k} \in W^{1, r}(\Omega) \quad(k=1, \ldots, N)
\end{aligned} \quad \text { where } \begin{cases}r=2 & \text { if } l=1 \\
r>2 & \text { if } l=2 \\
r=l & \text { if } l \geq 3 .\end{cases}
$$

Then,

$$
\begin{aligned}
A & \in \mathcal{L}(Y, X) \\
B_{k} & \in \mathcal{L}(Y, X) \quad(k=1, \ldots, N) .
\end{aligned}
$$

Moreover, $A$ is closed, self-adjoint and bounded from above in $X$. This implies that $A$ generates a cosine family in $X$ (see [9: p. 104]). The interpolation space associated with $X$ and $Y$ is $Y_{1}=H_{0}^{1}(\Omega)$ [9: p. 106].

We recall that abstract problem (3.3) treated in Sections 3 - 7 is a generalization of problem (2.11) obtained from (2.3) - (2.5), (2.8) by the change of unknown function (2.10). The assumptions of Lemma 5.1 are satisfied for the data $\widetilde{\varphi}, \widetilde{\psi}, \widetilde{f}, w, \widetilde{h}$ of problem (2.11) provided the following relations hold true:

$$
\left.\begin{array}{l}
\widetilde{\varphi}, \widetilde{\psi} \in H^{2}(\Omega) \cap H_{0}^{1}(\Omega) \\
\widetilde{f}=\widetilde{f}_{1}+1 * \widetilde{f}_{2}+t * \widetilde{f}_{3} \\
\widetilde{f_{1}}+\operatorname{div}(\lambda \nabla \widetilde{\varphi}) \in W^{1,1}\left((0, T) ; H^{2}(\Omega) \cap H_{0}^{1}(\Omega)\right) \\
\widetilde{f}_{2}+\operatorname{div}(\lambda \nabla \widetilde{\psi}) \in W^{1,1}\left((0, T) ; H_{0}^{1}(\Omega)\right), \widetilde{f}_{3} \in W^{3,1}\left((0, T) ; L_{2}(\Omega)\right) \\
w \in C^{2}\left([0, T] ;\left(L^{2}(\Omega)\right)^{N}\right) \\
\operatorname{div}\left(\mu_{k} \nabla \varphi\right) \in H_{0}^{1}(\Omega) \quad(k=1, \ldots, N) \\
\widetilde{h} \in H^{2}\left((0, T) ; \mathbb{R}^{N}\right), h(0)=\Psi[\varphi]
\end{array}\right\} .
$$

Next we mention that in the case $l \geq 2$ from (2.9) assumption (3.2), i.e. the relations

$$
\left.\begin{array}{rl}
\Psi_{i} & \in\left(H^{2}(\Omega)\right)^{*} \\
\Phi_{i k} & \in\left(H^{2}(\Omega)\right)^{*}
\end{array}\right\} \quad(i, k=1, \ldots, N)
$$

follow provided the functions $\lambda$ and $\mu_{k}$ fulfill the conditions

$$
\lambda, \mu_{k} \in C^{\sigma}(\bar{\Omega}) \quad(k=1, \ldots, N) \text { for some } \sigma \in\left(\frac{1}{2}, 1\right] .
$$

In the case $l=1$ in (2.9) relations (8.3) follow from conditions (8.1) above.

8.1 Existence, uniqueness and stability. Now we are ready to formulate an existence, uniqueness and stability theorem for problem (2.3) - (2.5), (2.8) simply applying Theorem 6.1 to equivalent problem (2.11). We are going to state this theorem for problem (2.3) - (2.5), (2.8) where $\Psi_{i}$ and $\Phi_{i k}$ are general 
functionals satisfying conditions (8.3) and need not to be of form (2.9). Then, imposing additional convergence conditions on these functionals, we are able to incorporate the non-degenerate case $N=+\infty$ in the theorem, too.

Although, as we will see later on, in the case $N=+\infty$ the theorem does not apply to the problems with functionals of form (2.9), we think that such a more general formulation could be useful in further applications.

Theorem 8.1. Let $N$ be a fixed integer in $\mathbb{N} \cup\{+\infty\}$ and $T \in(0,+\infty)$. Assume that conditions (8.1) - (8.3), (5.1) $)_{4}$ as well as (5.2) when $N=+\infty$ are satisfied for the operators $B, \Psi, \Phi$ and the set of data $d=(\varphi, \psi, \hat{u}, f, h)$. Moreover, let $\hat{u} \in C^{2}\left([0, T] ; H^{2}(\Omega)\right)$ and the condition

$$
\kappa[d]^{2}=\inf _{\xi \in \mathbb{R}^{N} \backslash\{0\}}\|\xi\|_{\mathbb{R}^{N}}^{-2} \sum_{i, k=1}^{N} \Phi_{i k}[\varphi] \xi_{i} \xi_{k}>0
$$

hold. Then identification problem (2.3) - (2.5), (2.8) has a unique solution $(m, u)$ in the space $\mathcal{S}_{\star}^{N}=H^{1}\left((0, T) ; \mathbb{R}^{N}\right) \times C^{2}\left([0, T] ; H^{2}(\Omega)\right)$. Moreover, the solutions $S^{1}=\left(m^{1}, u^{1}\right)$ and $S^{2}=\left(m^{2}, u^{2}\right)$ corresponding to two sets of data

$$
\begin{aligned}
& d^{1}=\left(\varphi^{1}, \psi^{1}, \hat{u}^{1}, f^{1}, h^{1}\right) \\
& d^{2}=\left(\varphi^{2}, \psi^{2}, \hat{u}^{2}, f^{2}, h^{2}\right)
\end{aligned}
$$

respectively, satisfy the stability estimate

$$
\left\|S^{1}-S^{2}\right\|_{N}^{\star} \leq K_{\star}\left(\omega_{N}, \kappa\left[d^{1}\right]^{-1}, \kappa\left[d^{2}\right]^{-1},\left|d^{1}\right|_{N}^{\star},\left|d^{2}\right|_{N}^{\star}\right)\left|d^{1}-d^{2}\right|_{N}^{\star}
$$

where

$$
\|S\|_{N}^{\star}=\|m\|_{H^{1}\left((0, T) ; \mathbb{R}^{N}\right)}+\|u\|_{C^{2}\left([0, T] ; H^{2}(\Omega)\right)}
$$

is the norm in $\mathcal{S}_{\star}^{N}, \omega_{N}$ is given by (6.5) and $K_{\star}$ is a function of the form $K=\exp \left(P^{6,3,3,2,2}\right)$ depending also on $T$. The seminorm $|\cdot|_{N}^{\star}$ is given by

$$
\begin{aligned}
|d|_{N}^{\star}= & \|\widetilde{\varphi}\|_{H^{2}(\Omega) \cap H_{0}^{1}(\Omega)}+\|\widetilde{\psi}\|_{H^{2}(\Omega) \cap H_{0}^{1}(\Omega)}+\|\hat{u}\|_{C^{2}\left([0, T] ; H^{2}(\Omega)\right)} \\
& +\left\|\widetilde{f}_{1}+\operatorname{div}(\lambda \nabla \widetilde{\varphi})\right\|_{W^{1,1}\left((0, T) ; H^{2}(\Omega) \cap H_{0}^{1}(\Omega)\right)} \\
& +\left\|\widetilde{f}_{2}+\operatorname{div}(\lambda \nabla \widetilde{\psi})\right\|_{W^{1,1}\left((0, T) ; H_{0}^{1}(\Omega)\right)} \\
& +\left\|\widetilde{f}_{3}\right\|_{W^{1,1}\left((0, T) ; L^{2}(\Omega)\right)}+\left\|D_{t} w\right\|_{C^{1}\left([0, T] ;\left(L^{2}(\Omega)\right)^{N}\right)} \\
& +\left(\sum_{k=1}^{N}\left\|\operatorname{div}\left(\mu_{k} \nabla \varphi\right)\right\|_{H_{0}^{1}(\Omega)}^{2}\right)^{1 / 2} \\
& +\left\|D_{t} \chi\right\|_{C^{1}\left([0, T] ; \mathcal{L}\left(\mathbb{R}^{N}, \mathbb{R}^{N}\right)\right)}+\left\|D_{t} \widetilde{h}\right\|_{H^{1}\left((0, T) ; \mathbb{R}^{N}\right)}
\end{aligned}
$$

and $\widetilde{\varphi}, \widetilde{\psi}, \widetilde{f}, w, \chi, \widetilde{h}$ are given in terms of the data d by formulae (2.12). 
Remark 8.1. If the set of data $d=(\varphi, \psi, \hat{u}, f, h)$ satisfies the assumptions in Theorem 8.1 with some $N \in \mathbb{N} \cup\{+\infty\}$ and every $T \in(0,+\infty)$, then identification problem $(2.3)-(2.5),(2.8)$, reformulated for $t \in(0,+\infty)$, admits a unique solution $(m, u)$ in the space $H_{l o c}^{1}\left((0,+\infty) ; \mathbb{R}^{N}\right) \times \bigcup_{T>0} C^{2}\left([0, T] ; H^{2}(\Omega)\right)$.

8.2 Approximation results. In this subsection we study problem (2.3) (2.5), (2.8) in the case $l \geq 2$, the functionals $\Psi_{i}$ and $\Phi_{i k}$ being of form (2.9). First we will show that such functionals $\Psi_{i}$ and $\Phi_{i k}$ do not meet the requirements of Theorem 8.1 in the case $N=+\infty$. However, Theorem 8.1 holds in the case $N<+\infty$ and we can show, using the arguments of Section 7 that the solution corresponding to finite $N$ converges to a solution corresponding to $N=+\infty$, assuming a priori the existence and sufficient regularity of the solution to the latter problem. Concerning the one-dimensional case $l=1$ we make only a short remark at the end of this subsection.

Let $l \geq 2$ and $\Psi_{i}$ and $\Phi_{i k} \quad(i, k=1, \ldots, N)$ be of form (2.9). If $\lambda$ and $\mu=\left(\mu_{k}\right)_{k=1, \ldots, N}$ satisfy $(8.4)$ and $\eta=\left(\eta_{i}\right)_{i=1, \ldots, N} \in H^{-1 / 2}\left(\Gamma ; \mathbb{R}^{N}\right)$, then conditions (8.3) hold. Further, using trace and embedding theorems, for any $\sigma \in\left(\frac{1}{2}, 1\right]$ we have

$$
\left.\begin{array}{rl}
\left\|B_{k}\right\| & \leq c_{1}\left\|\mu_{k}\right\|_{W^{1, r}(\Omega)} \\
\left\|\Psi_{i}\right\| & \leq c_{2}\left\|\eta_{i}\right\|_{H^{-1 / 2}(\Gamma)} \\
\left\|\Phi_{i k}\right\| & \leq c_{3}\left\|\eta_{i}\right\|_{H^{-1 / 2}(\Gamma)}\left(\left\|\mu_{k}\right\|_{W^{1, r}(\Omega)}+\left\|\mu_{k}\right\|_{C^{\sigma}(\bar{\Omega})}\right)
\end{array}\right\}
$$

for all $i, k=1, \ldots, N$ where the constants $c_{1}, c_{2}, c_{3}$ depend on $\Omega$ and $c_{2}$ on $\lambda$, too. Therefore, $\omega_{N}$ (cf. definition (6.5)) satisfies $\omega_{N} \leq \bar{\omega}_{N}$ where

$$
\begin{aligned}
\bar{\omega}_{N}= & c_{4}\left\{\|\eta\|_{H^{-1 / 2}\left(\Gamma ; \mathbb{R}^{N}\right)}+\|\mu\|_{W^{1, r}\left(\Omega ; \mathbb{R}^{N}\right)}+\|\mu\|_{C^{\sigma}\left(\bar{\Omega} ; \mathbb{R}^{N}\right)}\right. \\
& \left.+\|\eta\|_{H^{-1 / 2}\left(\Gamma ; \mathbb{R}^{N}\right)}\left(\|\mu\|_{W^{1, r}\left(\Omega ; \mathbb{R}^{N}\right)}+\|\mu\|_{C^{\sigma}\left(\bar{\Omega} ; \mathbb{R}^{N}\right)}\right)\right\},
\end{aligned}
$$

$c_{4}>0$ being a constant independent of $N$. If we assume

$$
\bar{\omega}_{N} \leq C \quad(N \in \mathbb{N})
$$

(this is a sufficient condition for (5.2)), then from (8.8) we immediately have

$$
\Phi_{i i}[\varphi]=\left\langle\eta_{i}, \mu_{i} D_{\nu} \varphi\right\rangle_{1 / 2, \Gamma} \rightarrow 0 \quad \text { as } i \rightarrow+\infty
$$

so that (8.5) fails for $N=+\infty$. Therefore, we cannot expect that Theorem 8.1 applies to problem $(2.3)-(2.5),(2.8)$ when $N=+\infty$ and the functionals $\Psi_{i}$ and $\Phi_{i k}$ are of form (2.9). 
Remark 8.2. Of course, there exist bases $\left(\mu_{k}\right)_{k \in \mathbb{N}}$ for which boundedness relation (8.10) holds. For instance, when $\Omega$ is an open bounded set in $\mathbb{R}^{l}$ of class $C^{2 l+4},\left(\mu_{k}\right)_{k \in \mathbb{N}}$ can chosen to be an orthogonal complete system in $L^{2}(\Omega)$ (cf. [23]) such that

$$
\left.\begin{array}{rl}
(-\Delta)^{l+2} \mu_{k}+\mu_{k} & =\rho_{k} \mu_{k} \quad \text { in } \Omega \\
D_{\nu}\left(\Delta^{j} \mu_{k}\right)_{\mid \Gamma} & =0 \quad(j=0,1, \ldots, l+1)
\end{array}\right\}
$$

and $(i, k \in \mathbb{N})$

$$
\left.\begin{array}{c}
\int_{\Omega}\left[\Delta^{(l+2) / 2} \mu_{i}(x) \cdot \Delta^{(l+2) / 2} \mu_{k}(x)+\mu_{i}(x) \mu_{k}(x)\right] d x=\delta_{i k} \quad\left(\frac{l}{2} \in \mathbb{N}\right) \\
\int_{\Omega}\left[\nabla \Delta^{(l+1) / 2} \mu_{i}(x) \cdot \nabla \Delta^{(l+1) / 2} \mu_{k}(x)+\mu_{i}(x) \mu_{k}(x)\right] d x=\delta_{i k} \quad\left(\frac{l+1}{2} \in \mathbb{N}\right) \\
\int_{\Omega} \mu_{i}(x) \mu_{k}(x) d x=\rho_{k}^{-1} \delta_{i k}
\end{array}\right\}
$$

$\left(\rho_{k}\right)_{k \in \mathbb{N}}$ and $\delta_{i k}$ being, respectively, a positive non-decreasing sequence diverging to $+\infty$ and the Kronecker delta.

If $\left(\lambda_{k}\right)_{k \in \mathbb{N}}$ and $\left(v_{k}\right)_{k \in \mathbb{N}}$ denote, respectively, the sequences of the eigenvalues and eigenfunctions related to the spectral problem

$$
\left.\begin{array}{rl}
-\Delta v & =\lambda v \text { in } \Omega \\
D_{\nu} v_{\mid \Gamma} & =0
\end{array}\right\}
$$

we see that $v_{k}$ is an eigenfunction of problem (8.11) and $\rho_{k}=1+\lambda_{k}^{l+2}$. Since $\lambda_{k} \sim c k^{2 / l}$ as $k \rightarrow+\infty$ (cf. [8: Chapter 6]), we easily deduce the inequalities

$$
0<\rho_{k} \leq C k^{-2(l+2) / l} \quad(k \in \mathbb{N}) .
$$

Since

$$
u \rightarrow\left\{\begin{array}{l}
\left(\int_{\Omega}\left[\left|\Delta^{(n+2) / 2]} u(x)\right|^{2}+|u(x)|^{2}\right] d x\right)^{1 / 2} \\
\left(\int_{\Omega}\left[\left|\nabla \Delta^{(n+1) / 2} u(x)\right|^{2}+|u(x)|^{2}\right] d x\right)^{1 / 2}
\end{array}\right.
$$

are norms in $H^{n+2}(\Omega)$ equivalent to the usual one, from (8.12) we derive the estimates

$$
\left.\begin{array}{rl}
\left\|\mu_{k}\right\|_{L^{2}(\Omega)} & \leq C_{0} k^{-(l+2) / l} \\
\left\|\mu_{k}\right\|_{H^{l+2}(\Omega)} & \leq C_{1}
\end{array}\right\} \quad(k \in \mathbb{N}) .
$$

They imply, via Sobolev embedding theorems, the continuous embedding

$$
H^{n_{0}+1}(\Omega) \hookrightarrow W^{1, r}(\Omega) \cap C^{\sigma}(\bar{\Omega}) \quad \text { for some } \sigma \in\left(\frac{1}{2}, 1\right)
$$


and the inequalities

$$
\left\|\mu_{k}\right\|_{W^{1, r}(\Omega)}+\left\|\mu_{k}\right\|_{C^{\sigma}(\bar{\Omega})} \leq C_{2}\left\|\mu_{k}\right\|_{H^{n_{0}+1}(\Omega)} \quad(k \in \mathbb{N})
$$

where

$$
\begin{cases}r=2 & \text { if } l=1 \\ r \in(2,+\infty) & \text { if } l=2 \\ r=l & \text { if } l \geq 3\end{cases}
$$

and $n_{0}=\left[\frac{l+1}{2}\right]$ with $[x]$ denoting the largest integer not exceeding $x$.

Using (8.13) and well-known interpolation properties, we derive the estimates

$$
\left\|\mu_{k}\right\|_{H^{n_{0}+1}(\Omega)} \leq C_{3} k^{-\left(l+1-n_{0}\right) / l} \quad(k \in \mathbb{N}) .
$$

Consequently, since $\frac{l+1-n_{0}}{l}>\frac{1}{2}$, we conclude that $\sum_{k=1}^{+\infty}\left\|\mu_{k}\right\|_{H^{n_{0}+1}(\Omega)}^{2}<+\infty$. Finally, from (8.9) we derive the inequalities

$$
\bar{\omega}_{N} \leq C_{4}\left(1+\|\eta\|_{\left(H^{-1 / 2}(\Gamma)\right)^{\infty}}\right) \quad(N \in \mathbb{N})
$$

guaranteeing the boundedness of $\left(\bar{\omega}_{N}\right)_{N \in \mathbb{N}}$ provided the weights of the measurements $\eta_{i}$ are chosen so as to satisfy $\|\eta\|_{\left(H^{-1 / 2}(\Gamma)\right)^{\infty}}<+\infty$.

Remark 8.3. Assume that $\left(\mu_{k}\right)_{k \in \mathbb{N}}$ is defined as in Remark 8.2. The sufficient conditions for $(8.2)_{6}$ to hold are:

$$
\varphi \in H^{3}(\Omega),\left.\quad \varphi\right|_{\Gamma}=\text { const },\left.\quad \Delta \varphi\right|_{\Gamma}=0 .
$$

Indeed, (8.13) and (8.14) imply $\operatorname{div}\left(\mu_{k} \nabla \varphi\right) \in H^{1}(\Omega)$. Further, due to $\left.\Delta \varphi\right|_{\Gamma}=$ 0 , we have

$$
\left.\operatorname{div}\left(\mu_{k} \nabla \varphi\right)\right|_{\Gamma}=\left.\nabla \mu_{k} \cdot \nabla \varphi\right|_{\Gamma} \quad(k=1, \ldots, N) .
$$

But the right-hand side in (8.15) equals zero due to the assumptions $\left.\varphi\right|_{\Gamma}=$ const and $\left.D_{\nu} \mu_{k}\right|_{\Gamma}=0$ (cf. $(8.11)$ ).

Now, making use of the arguments preceding Theorem 7.1, we are able to deduce the convergence of the solutions corresponding to finite $N$ 's to a solution $S^{\infty}=\left(m^{\infty}, u^{\infty}\right)$ corresponding to $N=+\infty$ if we a priori assume existence and sufficient regularity of $S^{\infty}$. For, let

$$
d^{\infty}=\left(\varphi^{\infty}, \psi^{\infty}, \widehat{u}^{\infty}, f^{\infty}, h^{\infty}\right) \quad \text { with } h^{\infty}=\left(h_{i}^{\infty}\right)_{i=1, \ldots, \infty}
$$

be a given data vector. Let us define the projector $\bar{P}^{n}$ by

$$
\bar{P}^{n} d^{\infty}=\left(\varphi^{\infty}, \psi^{\infty}, \widehat{u}^{\infty}, f^{\infty},\left(h_{i}^{\infty}\right)_{i=1, \ldots, n}\right)
$$


Assume that $\left|d^{\infty}\right|_{\infty}^{\star}<\infty$ and $\bar{P}^{n} d^{\infty}$ satisfy the conditions of Theorem 8.1 for each finite $n$. This in particular implies

$$
\kappa_{n}\left[d^{\infty}\right]^{2}=\inf _{\xi \in \mathbb{R}^{n} \backslash\{0\}}\|\xi\|_{\mathbb{R}^{n}}^{-2} \sum_{i, k=1}^{n} \xi_{i} \xi_{k}\left\langle\eta_{i}, \mu_{k} D_{\nu} \varphi^{\infty}\right\rangle_{1 / 2, \Gamma}>0
$$

for all $n \in \mathbb{N}$. Let us denote by $S^{n}=\left(m^{n}, u^{n}\right)$ the solution of problem $(2.3)$ - (2.5), (2.8) corresponding to $N=n$ and the data $\bar{P}^{n} d^{\infty}$. Further, suppose that problem $(2.3)-(2.5),(2.8)$ corresponding to $N=+\infty$ and the data $d^{\infty}$ has a solution $S^{\infty}=\left(m^{\infty}, u^{\infty}\right)$ such that $\bar{Q}^{n} S^{\infty}=\left(\left(m_{k}^{\infty}\right)_{k=1, \ldots, n}, u^{\infty}\right)$ belongs to $\mathcal{S}_{\star}^{n}$ for each finite $n$. We observe that $\bar{Q}^{n} S^{\infty}$ satisfies problem (2.3) - (2.5), (2.8) with $N=n$ and data

$$
\widetilde{d}^{n}=\bar{P}^{n} d^{\infty}+\delta^{n}
$$

where

$$
\begin{aligned}
\delta^{n} & =\left(0,0,0, \widehat{f}^{n, \infty},\left(\widehat{h}_{i}^{n, \infty}\right)_{i=1, \ldots, n}\right) \\
\widehat{f}^{n, \infty}(t, x) & =-\sum_{k=n+1}^{\infty} m_{k}^{\infty} * \operatorname{div}\left(\mu_{k}(x) \nabla u^{\infty}(t, x)\right) \\
\widehat{h}_{i}^{n, \infty}(t) & =\sum_{k=n+1}^{\infty} m_{k}^{\infty} *\left\langle\eta_{i}, \mu_{k} D_{\nu} u^{\infty}(t, \cdot)\right\rangle_{1 / 2, \Gamma} .
\end{aligned}
$$

We will make use of the decomposition

$$
\widehat{f}^{n, \infty}=1 * \widehat{f}_{2}^{n, \infty}+t * \widehat{f}_{3}^{n, \infty} \text { with }\left\{\begin{array}{l}
\widehat{f}_{2}^{n, \infty} \in W^{1,1}\left((0, T) ; H_{0}^{1}(\Omega)\right) \\
\widehat{f}_{3}^{n, \infty} \in W^{1,1}\left((0, T) ; L^{2}(\Omega)\right)
\end{array}\right.
$$

similar to that used in the proof of Theorem 7.2. Then, from the definition of the seminorm $|\cdot|_{n}^{\star}$ we have

$$
\begin{aligned}
& \left|\delta^{n}\right|_{n}^{\star}=\left\|\sum_{k=n+1}^{\infty} m_{k}^{\infty} \operatorname{div}\left(\mu_{k} \nabla \varphi^{\infty}\right)\right\|_{W^{1,1}\left((0, T) ; H_{0}^{1}(\Omega)\right)} \\
& +\left\|\sum_{k=n+1}^{\infty}\left[m_{k}^{\infty}(0) \operatorname{div}\left(\mu_{k} \nabla D_{t} u^{\infty}\right)+D_{t} m_{k}^{\infty} * \operatorname{div}\left(\mu_{k} \nabla D_{t} u^{\infty}\right)\right]\right\|_{W^{1,1}\left((0, T) ; L^{2}(\Omega)\right)} \\
& +\left\{\sum_{i=1}^{n}\left\|\sum_{k=n+1}^{\infty}\left[m_{k}^{\infty}\left\langle\eta_{i}, \mu_{k} D_{\nu} \varphi^{\infty}\right\rangle_{1 / 2, \Gamma}+m_{k}^{\infty} *\left\langle\eta_{i}, \mu_{k} D_{\nu} D_{t} u^{\infty}\right\rangle_{1 / 2, \Gamma}\right]\right\|_{H^{1}(0, T)}\right\}^{1 / 2} .
\end{aligned}
$$

Using the relations

$$
\begin{aligned}
\omega_{n} & \leq \bar{\omega}_{n} \\
\left|\bar{P}^{n} d^{\infty}\right|_{n}^{\star} & \leq\left|d^{\infty}\right|_{\infty}^{\star} \\
\left|\widetilde{d}^{n}\right|_{n}^{\star}=\left|\bar{P}^{n} d^{\infty}\right|_{n}^{\star}+\left|\delta^{n}\right|_{n}^{\star} & \leq\left|d^{\infty}\right|_{\infty}^{\star}+\left|\delta^{n}\right|_{n}^{\star} \\
\left|\bar{P}^{n} d^{\infty}-\widetilde{d}^{n}\right|_{n}^{\infty} & =\left|\delta^{n}\right|_{n}^{\star}
\end{aligned}
$$


and Theorem 8.1, we obtain the estimate

$$
\begin{aligned}
\| S^{n} & -\bar{Q}^{n} S^{\infty} \|_{n}^{\star} \\
& \leq K_{\star}\left(\bar{\omega}_{n}, \kappa_{n}\left[d^{\infty}\right]^{-1}, \kappa_{n}\left[d^{\infty}\right]^{-1},\left|d^{\infty}\right|_{\infty}^{\star},\left|d^{\infty}\right|_{\infty}^{\star}+\left|\delta^{n}\right|_{n}^{\star}\right)\left|\delta^{n}\right|_{n}^{\star}
\end{aligned}
$$

where $\bar{\omega}_{n}, \kappa_{n}\left[d^{\infty}\right]$ and $\left|\delta^{n}\right|_{n}^{\star}$ are given by (8.9), (8.16) and (8.17), respectively. If we suppose that the first argument $\bar{\omega}_{n}$ of $K_{\star}$ is bounded as $n \rightarrow+\infty$, then

$$
\left\langle\eta_{i}, \mu_{i} D_{\nu} \varphi^{\infty}\right\rangle_{1 / 2, \Gamma} \rightarrow 0 \quad \text { as } i \rightarrow+\infty
$$

and from (8.16) it follows $\kappa_{n}\left[d^{\infty}\right] \rightarrow 0$ as $n \rightarrow+\infty$. Hence the coefficient $K_{\star}$ increases as $n \rightarrow+\infty$. Nevertheless, the relation $\left\|S^{n}-\bar{Q}^{n} S^{\infty}\right\|_{n}^{\star} \rightarrow 0$ as $n \rightarrow+\infty$ holds whenever the solution $S^{\infty}=\left(m^{\infty}, u^{\infty}\right)$ is regular enough to guarantee the convergence $\left|\delta^{n}\right|_{n}^{\star} \rightarrow 0$ with a speed which compensates the increase of the factor $K_{\star}$ in (8.18). As a consequence of this statement, reasoning as in Corollary 7.1, we can deduce the uniqueness of the solution $S^{\infty}$ satisfying such a regularity property.

Remark 8.4. In the particular case when $\eta_{i}, \mu_{k}$ and $\varphi^{\infty}$ satisfy the relations

$$
\sum_{j=1, j \neq i}^{N}\left|\left\langle\eta_{i}, \mu_{j} D_{\nu} \varphi^{\infty}\right\rangle_{1 / 2, \Gamma}\right| \leq \varepsilon\left\langle\eta_{i}, \mu_{i} D_{\nu} \varphi^{\infty}\right\rangle_{1 / 2, \Gamma}
$$

with some $\varepsilon \in(0,1)$, we have the lower estimate

$$
\kappa_{n}\left[d^{\infty}\right]^{2} \geq(1-\varepsilon) \min _{1 \leq i \leq n}\left\langle\eta_{i}, \mu_{i} D_{\nu} \varphi^{\infty}\right\rangle_{1 / 2, \Gamma}
$$

for $\kappa_{n}\left[d^{\infty}\right]^{2}$.

Remark 8.5. The results of this subsection remain valid - with a few minor modifications - in the one-dimensional case $l=1, \Omega=(0,1)$ when the functionals $\Psi_{i}, \Phi_{i k}$ are of form (2.9). Then additional assumption (8.4) for $\lambda$ and $\mu_{k}$ is useless and the corresponding norms $\left\|\mu_{k}\right\|_{C^{\sigma}(\bar{\Omega})}$ and $\|\mu\|_{C^{\sigma}\left(\bar{\Omega} ; \mathbb{R}^{N}\right)}$ as well as the space $C^{\sigma}(\bar{\Omega})$ may be removed from formulae $(8.8)$ - (8.9) and the context of Remark 8.2.

As far as the weights $\left(\eta_{i}\right)_{i=1, \ldots, N}$ are concerned, we assume $\eta \in \mathbb{R}^{N}$ instead of $\eta \in H^{-1 / 2}\left(\Gamma ; \mathbb{R}^{N}\right)$. Moreover, the norm $\|\eta\|_{H^{-1 / 2}\left(\Gamma ; \mathbb{R}^{N}\right)}$ and the pairing $\left\langle\eta_{i}, v\right\rangle_{1 / 2, \Gamma}$ between $\eta_{i}$ and $v \in H^{1}(\Omega) \subset H^{1 / 2}(\Gamma)$, appearing in computations, have to be replaced by the norm $\|\eta\|_{\mathbb{R}^{N}}$ and the product $\eta_{i} v\left(x_{i}\right)$ for $v \in$ $H^{1}(0,1) \subset C[0,1] \quad\left(x_{i} \in[0,1]\right)$, respectively. In particular, boundedness relation (8.10) is satisfied for $\mu$ chosen as in Remark 8.2 provided the set of weights $\eta=\left(\eta_{i}\right)_{i \in \mathbb{N}}$ belongs to the space $\mathbb{R}^{\infty}=l_{2}$. 


\section{Proof of Lemma 6.1}

Taking advantage of the obvious relation

$$
e^{-\gamma t}\left(v_{1} * v_{2}\right)=\left(e^{-\gamma t} v_{1}\right) *\left(e^{-\gamma t} v_{2}\right)
$$

from (6.9) and (6.11) we derive the equalities

$$
\begin{aligned}
& e^{-\gamma t}(F[d] U)_{0}(t) \\
& =-\sum_{k=1}^{N}\left[m_{k}^{0}+\left(e^{-\gamma t} U_{k}\right) *\right]\left[\left(e^{-\gamma t} t * \mathcal{C}\right) *\left(e^{-\gamma t} B_{k} U_{0}(t)+e^{-\gamma t} w_{k}^{\prime \prime}(t)\right)\right. \\
& \left.\quad+e^{-\gamma t} t * \mathcal{C}(t)\left(B_{k} \psi+w_{k}^{\prime}(0)\right)+e^{-\gamma t} 1 * \mathcal{C}(t)\left(B_{k} \varphi+w_{k}(0)\right)\right]+e^{-\gamma t} \alpha(t)
\end{aligned}
$$

and, for $i=1, \ldots, N$,

$$
\begin{aligned}
e^{-\gamma t}(G[d] U)_{i}(t) \\
=-\sum_{k=1}^{N}\left\{( e ^ { - \gamma t } U _ { k } ) * \left[e^{-\gamma t} *\left(\Phi_{i k}\left[e^{-\gamma t} U_{0}(t)\right]+e^{-\gamma t} \chi_{i k}^{\prime \prime}(t)\right)\right.\right. \\
\left.\left.\quad+e^{-\gamma t}\left(\Phi_{i k}[\psi]+\chi_{i k}^{\prime}(0)\right)\right]+\left(e^{-\gamma t} m_{k}^{0}[d]\right) *\left(\Phi_{i k}\left[e^{-\gamma t} U_{0}(t)\right]+\chi_{i k}^{\prime \prime}(t)\right)\right\} \\
\quad+\Psi_{i}\left[e^{-\gamma t}(F[d] U)_{0}(t)\right]-e^{-\gamma t} h_{i}^{\prime \prime}(t)-\sum_{k=1}^{N} e^{-\gamma t} m_{k}^{0}[d]\left(\Phi_{i k}[\psi]+\chi_{i k}^{\prime}(0)\right)
\end{aligned}
$$

In order to estimate the right-hand sides in relations (9.1) and (9.2) we need the Young inequalities

$$
\begin{array}{cl}
\left\|v_{1} * v_{2}\right\|_{C([0, T] ; Y)} \leq\left\|v_{1}\right\|_{L^{1}(0, T)}\left\|v_{2}\right\|_{C([0, T] ; Y)} & \left\{\begin{array}{l}
v_{1} \in L^{1}(0, T) \\
v_{2} \in C([0, T] ; Y)
\end{array}\right. \\
\left\|v_{1} * v_{2}\right\|_{C([0, T] ; Y)} \leq\left\|v_{1}\right\|_{C[0, T]}\left\|v_{2}\right\|_{L^{1}((0, T) ; Y)} & \left\{\begin{array}{l}
v_{1} \in C[0, T] \\
v_{2} \in L^{1}((0, T) ; Y)
\end{array}\right. \\
\left\|v_{1} * v_{2}\right\|_{L^{p}(0, T)} \leq\left\|v_{1}\right\|_{L^{1}(0, T)}\left\|v_{2}\right\|_{L^{p}(0, T)} & \left\{\begin{array}{l}
v_{1} \in L^{1}(0, T) \\
v_{2} \in L^{p}(0, T)
\end{array}\right.
\end{array}
$$

Moreover, from Lemma 4.1 and the uniform boundedness principle we deduce the estimates

$$
\left.\begin{array}{rl}
\sup _{0 \leq t \leq T}\|\mathcal{C}(t)\|_{\mathcal{L}(Y)} & \leq C_{1} \\
\sup _{0 \leq t \leq T}\|1 * \mathcal{C}(t)\|_{\mathcal{L}\left(Y_{1}, Y\right)} & \leq C_{2} \\
\sup _{0 \leq t \leq T}\|t * \mathcal{C}(t)\|_{\mathcal{L}(X, Y)} \leq C_{3}
\end{array}\right\}
$$


for some constants $C_{1}, C_{2}$ and $C_{3}$ depending on $T$.

In addition to the weighted norm $\|\cdot\|_{2, \gamma}$ defined in $L^{2}(0, T)$ by $(6.13)_{2}$, let us introduce similar norms in $L^{p}(0, T) \quad(p \in[0, \infty])$ by

$$
\|v\|_{p, \gamma}=\left\|e^{-\gamma t} v\right\|_{L^{p}(0, T)} \quad\left(v \in L^{p}(0, T), \gamma \geq 0\right) .
$$

We can now estimate $F[d] U$ and $G[d] U$. Making use of (9.3) - (9.6), we obtain

$$
\begin{aligned}
&\left\|(F[d] U)_{0}\right\|_{\infty, \gamma} \\
& \leq \sum_{k=1}^{N}\left\{\left[\left|m_{k}^{0}[d]\right|+\left\|U_{k}\right\|_{1, \gamma}\right]\|1\|_{1, \gamma} C_{3}\right. \\
& \times\left(\left\|B_{k}\right\|\left\|U_{0}\right\|_{\infty, \gamma}+\left\|w^{\prime \prime}\right\|_{C([0, T] ; X)}\right)+\left[\left|m_{k}^{0}[d]\right|+\left\|U_{k}\right\|_{2, \gamma}\|1\|_{2, \gamma}\right] \\
&\left.\times\left[C_{3}\left(\left\|B_{k}\right\|\|\psi\|_{Y}+\left\|w_{k}^{\prime}(0)\right\|_{X}\right)+C_{2}\left(\left\|B_{k} \varphi\right\|_{Y_{1}}+\left\|w_{k}(0)\right\|_{Y_{1}}\right)\right]\right\} \\
&+\max _{0 \leq t \leq T}\|\alpha(t)\|_{Y}
\end{aligned}
$$

and, for $i=1, \ldots, N$,

$$
\begin{aligned}
& \left\|(G[d] U)_{i}\right\|_{2, \gamma} \\
& \leq \sum_{k=1}^{N}\|1\|_{1, \gamma}\left\{\| U _ { k } \| _ { 2 , \gamma } \left(T\left\|\Phi_{i k}\right\|\left\|U_{0}\right\|_{\infty, \gamma}+T\left\|\chi_{i k}^{\prime \prime}\right\|_{C[0, T]}\right.\right. \\
& \left.\left.\quad+\left\|\Phi_{i k}\right\|\|\psi\|_{Y}+\left|\chi_{i k}^{\prime}(0)\right|\right)+\left|m_{k}^{0}[d]\right| T^{1 / 2}\left(\left\|\Phi_{i k}\right\|\left\|U_{0}\right\|_{\infty, \gamma}+\left\|\chi_{i k}^{\prime \prime}\right\|_{C[0, T]}\right)\right\} \\
& \quad+T^{1 / 2}\left\|\Psi_{i}\right\|\left\|(F[d] U)_{0}\right\|_{\infty, \gamma}+\left\|h_{i}^{\prime \prime}\right\|_{L^{2}(0, T)} \\
& \quad+\sum_{k=1}^{N} T^{1 / 2}\left|m_{k}^{0}[d]\right|\left(\left\|\Phi_{i k}\right\|\|\psi\|_{Y}+\left|\chi_{i k}^{\prime}(0)\right|\right) .
\end{aligned}
$$

Due to definition (5.4) of $\alpha$ and (6.4) of $|d|_{N}$, making use of the inequality

$$
\|1\|_{r, \gamma} \leq\left(\frac{1}{r \gamma}\right)^{-1 / r} \quad(\gamma \geq 0,1 \leq r<+\infty)
$$

and relations (9.6) we get

$$
\max _{0 \leq t \leq T}\|\alpha(t)\|_{X} \leq C_{4}|d|_{N}
$$

for some constant $C_{4}>0$ depending on $T$. 
Taking estimates (6.6) and (9.9) - (9.10) into account, from (9.7) - (9.8) we obtain

$$
\begin{aligned}
& \left\|(F[d] U)_{0}\right\|_{\infty, \gamma} \\
& \quad \leq P^{2,1,2}\left(\omega_{N}, \kappa[d]^{-1},|d|_{N}\right)\left(1+\gamma^{-1 / 2}\|U\|_{\gamma}\right)+P^{1}\left(\omega_{N}\right) \gamma^{-1}\|U\|_{\gamma}^{2}
\end{aligned}
$$

and

$$
\begin{aligned}
\left(\sum_{i=1}^{N}\right. & \left.\left\|(G[d] U)_{i}\right\|_{2, \gamma}^{2}\right)^{1 / 2} \\
\leq & P^{2,1,2}\left(\omega_{N}, \kappa[d]^{-1},|d|_{N}\right)\left(1+\gamma^{-1}\|U\|_{\gamma}\right) \\
& +P^{1}\left(\omega_{N}\right) \gamma^{-1}\|U\|_{\gamma}^{2}+\omega_{N}\left\|(F[d] U)_{0}\right\|_{\infty, \gamma} \\
\leq & P^{3,1,2}\left(\omega_{N}, \kappa[d]^{-1},|d|_{N}\right)\left(1+\gamma^{-1 / 2}\|U\|_{\gamma}\right)+P^{2}\left(\omega_{N}\right) \gamma^{-1}\|U\|_{\gamma}^{2} .
\end{aligned}
$$

Estimating (6.10) by means of (9.12) and taking (6.1) into account, we obtain

$$
\begin{aligned}
& \left(\sum_{i=1}^{N}\left\|(F[d] U)_{i}\right\|_{2, \gamma}^{2}\right)^{1 / 2} \\
& \quad \leq P^{3,2,2}\left(\omega_{N}, \kappa[d]^{-1},|d|_{N}\right)\left(1+\gamma^{-1 / 2}\|U\|_{\gamma}\right)+P^{2,1}\left(\omega_{N}, \kappa[d]^{-1}\right) \frac{1}{\gamma}\|U\|_{\gamma}^{2} .
\end{aligned}
$$

Combining (9.11) and (9.13), we obtain estimate (6.14) for $F$.

Proceeding likewise, we can derive a Lipschitz-type estimate for $F$. Let

$$
d^{i}=\left(\varphi^{i}, \psi^{i}, f^{i}=f_{1}^{i}+1 * f_{2}^{i}+t * f_{3}^{i}, h^{i}\right) \quad(i=1,2)
$$

be two data sets. For $i=1,2$ we define $\alpha^{i}$ by means of (5.4) and $\varphi^{i}, \psi^{i}, f_{1}^{i}, f_{2}^{i}, f_{3}^{i}$. From (6.9) - (6.11) we obtain the relations

$$
\begin{aligned}
\left(F\left[d^{1}\right]\right. & \left.U^{1}-F\left[d^{2}\right] U^{2}\right)_{0}(t) \\
= & -\sum_{k=1}^{N}\left\{\left[m_{k}^{0}\left[d^{1}\right]-m_{k}^{0}\left[d^{2}\right]+\left(U_{k}^{1}-U_{k}^{2}\right) *\right]\right. \\
& \times\left[t * \mathcal{C} *\left(B_{k} U_{0}^{1}(t)+D_{t}^{2} w_{k}^{1}(t)\right)+t * \mathcal{C}(t)\left(B_{k} \psi^{1}+D_{t} w_{k}^{1}(0)\right)\right. \\
& \left.+1 * \mathcal{C}(t)\left(B_{k} \varphi^{1}+w_{k}^{1}(0)\right)\right]+\left[m_{k}^{0}\left[d^{2}\right]+U_{k}^{2} *\right] \\
& \times\left[t * \mathcal{C} *\left(B_{k}\left(U_{0}^{1}(t)-U_{0}^{2}(t)\right)+D_{t}^{2}\left(w_{k}^{1}-w_{k}^{2}\right)(t)\right)\right. \\
& +t * \mathcal{C}(t)\left(B_{k}\left(\psi^{1}-\psi^{2}\right)+D_{t}\left(w_{k}^{1}-w_{k}^{2}\right)(0)\right) \\
& \left.\left.+1 * \mathcal{C}(t)\left(B_{k}\left(\varphi^{1}-\varphi^{2}\right)+\left(w_{k}^{1}-w_{k}^{2}\right)(0)\right)\right]\right\}+\alpha^{1}(t)-\alpha^{2}(t),
\end{aligned}
$$


further

$$
\begin{aligned}
& \sum_{k=1}^{N}\left(\mid \Phi_{i k}\left[\varphi^{1}\right]+\chi_{i k}^{1}(0)\right)\left(F\left[d^{1}\right] U^{1}-F\left[d^{2}\right] U^{2}\right)_{k}(t) \\
& =\sum_{k=1}^{N}\left(\mid \Phi_{i k}\left[\varphi^{2}-\varphi^{1}\right]+\chi_{i k}^{2}(0)-\chi_{i k}^{1}(0)\right)\left(F\left[d^{2}\right] U^{2}\right)_{k}(t) \\
& \quad+\left(G\left[d^{1}\right] U^{1}-G\left[d^{2}\right] U^{2}\right)_{i}(t) \quad(i=1, \ldots, N)
\end{aligned}
$$

and at last

$$
\begin{aligned}
\left(G\left[d^{1}\right]\right. & \left.U^{1}-G\left[d^{2}\right] U^{2}\right)_{i}(t) \\
= & -\sum_{k=1}^{N}\left\{\left(U_{k}^{1}-U_{k}^{2}\right) * 1 *\left(\Phi_{i k}\left[U_{0}^{1}(t)\right]+D_{t}^{2} \chi_{i k}^{1}(t)\right)\right. \\
& +U_{k}^{2} * 1 *\left(\Phi_{i k}\left[\left(U_{0}^{1}-U_{0}^{2}\right)(t)\right]+D_{t}^{2}\left(\chi_{i k}^{1}-\chi_{i k}^{2}\right)(t)\right) \\
& +\left(U_{k}^{1}-U_{k}^{2}\right) *\left(\Phi_{i k}\left[\psi^{1}\right]+D_{t} \chi_{i k}^{1}(0)\right) \\
& +U_{k}^{2} *\left(\Phi_{i k}\left[\psi^{1}-\psi^{2}\right]+D_{t}\left(\chi_{i k}^{1}-\chi_{i k}^{2}\right)(0)\right) \\
& +\left(m_{k}^{0}\left[d^{1}\right]-m_{k}^{0}\left[d^{2}\right]\right) *\left(\Phi_{i k}\left[U_{0}^{1}(t)\right]+D_{t}^{2} \chi_{i k}^{1}(t)\right) \\
& \left.+m_{k}^{0}\left[d^{2}\right] *\left(\Phi_{i k}\left[\left(U_{0}^{1}-U_{0}^{2}\right)(t)\right]+D_{t}^{2}\left(\chi_{i k}^{1}-\chi_{i k}^{2}\right)(t)\right)\right\} \\
& +\Psi_{i}\left[\left(F\left[d^{1}\right] U^{1}-F\left[d^{2}\right] U^{2}\right)_{0}(t)\right]-D_{t}^{2}\left(h_{i}^{1}-h_{i}^{2}\right)(t) \\
& -\sum_{k=1}^{N}\left\{\left(m_{k}^{0}\left[d^{1}\right]-m_{k}^{0}\left[d^{2}\right]\right)\left(\Phi_{i k}\left[\psi^{1}\right]+D_{t} \chi_{i k}^{1}(0)\right)\right. \\
& \left.+m_{k}^{0}\left[d^{2}\right]\left(\Phi_{i k}\left[\psi^{1}-\psi^{2}\right]+D_{t}\left(\chi_{i k}^{1}-\chi_{i k}^{2}\right)(0)\right)\right\}(i=1, \ldots, N) .
\end{aligned}
$$

We can estimate the right-hand sides in relations (9.14) and (9.16) similarly to relations (6.9) and (6.11). Performing long but simple computations, by means of inequalities (9.3) - (9.6), (9.10) as well as of (6.6) - (6.7), from (9.14) and (9.16) we derive the estimates

$$
\begin{aligned}
\|(F[ & \left.\left.d^{1}\right] U^{1}-F\left[d^{2}\right] U^{2}\right)_{0} \|_{\infty, \gamma} \\
\leq & P^{3,1,1,1,1,1,1}\left(\omega_{N}, \kappa\left[d^{1}\right]^{-1}, \kappa\left[d^{2}\right]^{-1},\left|d^{1}\right|_{N},\left|d^{2}\right|_{N},\left\|U^{1}\right\|_{\gamma},\left\|U^{2}\right\|_{\gamma}\right)\left|d^{1}-d^{2}\right|_{N} \\
& +\left\{P^{2,1,1,1}\left(\omega_{N}, \kappa\left[d^{2}\right]^{-1},\left|d^{1}\right|_{N},\left|d^{2}\right|_{N}\right)+P^{1}\left(\omega_{N}\right)\left(\left\|U^{1}\right\|_{\gamma}+\left\|U^{2}\right\|_{\gamma}\right)\right\} \\
& \times \gamma^{-1 / 2}\left\|U^{1}-U^{2}\right\|_{\gamma}
\end{aligned}
$$


and

$$
\begin{aligned}
& \left(\sum_{i=1}^{N}\left\|\left(G\left[d^{1}\right] U^{1}-G\left[d^{2}\right] U^{2}\right)_{i}\right\|_{2, \gamma}^{2}\right)^{1 / 2} \\
& \leq P^{3,1,1,1,1,1,1}\left(\omega_{N}, \kappa\left[d^{1}\right]^{-1}, \kappa\left[d^{2}\right]^{-1},\left|d^{1}\right|_{N},\left|d^{2}\right|_{N},\left\|U^{1}\right\|_{\gamma},\left\|U^{2}\right\|_{\gamma}\right)\left|d^{1}-d^{2}\right|_{N} \\
& \quad+\left\{P^{2,1,1,1}\left(\omega_{N}, \kappa\left[d^{2}\right]^{-1},\left|d^{1}\right|_{N},\left|d^{2}\right|_{N}\right)+P^{1}\left(\omega_{N}\right)\left(\left\|U^{1}\right\|_{\gamma}+\left\|U^{2}\right\|_{\gamma}\right)\right\} \\
& \quad \times \gamma^{-1 / 2}\left\|U^{1}-U^{2}\right\|_{\gamma}+\omega_{N}\left\|\left(F\left[d^{1}\right] U^{1}-F\left[d^{2}\right] U^{2}\right)_{0}\right\|_{\infty, \gamma} .
\end{aligned}
$$

Inserting (9.17) into (9.18), we derive an estimate similar to (9.18), but without the term $\left\|\left(F\left[d^{1}\right] U^{1}-F\left[d^{2}\right] U^{2}\right)_{0}\right\|_{\infty, \gamma}$. Further, applying this estimate, (6.1) and (9.13) in (9.15), we obtain

$$
\begin{aligned}
& \left(\sum_{i=1}^{N}\left\|\left(F\left[d^{1}\right] U^{1}-F\left[d^{2}\right] U^{2}\right)_{i}\right\|_{2, \gamma}^{2}\right)^{1 / 2} \\
& \leq \leq P^{6,2,2,1,2,1,2}\left(\omega_{N}, \kappa\left[d^{1}\right]^{-1}, \kappa\left[d^{2}\right]^{-1},\left|d^{1}\right|_{N},\left|d^{2}\right|_{N},\left\|U^{1}\right\|_{\gamma},\left\|U^{2}\right\|_{\gamma}\right)\left|d^{1}-d^{2}\right|_{N} \\
& \quad+\left\{P^{4,1,1,1,1}\left(\omega_{N}, \kappa\left[d^{1}\right]^{-1}, \kappa\left[d^{2}\right]^{-1},\left|d^{1}\right|_{N},\left|d^{2}\right|_{N}\right)\right. \\
& \left.\quad+P^{3,1}\left(\omega_{N}, \kappa\left[d^{1}\right]^{-1}\right)\left(\left\|U^{1}\right\|_{\gamma}+\left\|U^{2}\right\|_{\gamma}\right)\right\} \gamma^{-1 / 2}\left\|U^{1}-U^{2}\right\|_{\gamma}
\end{aligned}
$$

Finally, (9.17) and (9.19) yield (6.15)

\section{References}

[1] Bukhgeim, A. L., Dyatlov, G. V. and V. Isakov: Stability of memory reconstruction from the Dirichlet-to-Neumann operator. Siberian Math. J. 38 (1997), 636 - 646 .

[2] Bukhgeim, A. L. and G. V. Dyatlov: Inverse problems for equations with memory. Inverse Problems and Related Topics. Chapman \& Hall/CRC, Res. Notes 419 (2000), $19-35$.

[3] Cavaterra, C.: An inverse problem for a viscoelastic Timoshenko beam model. Z. Anal. Anw. 17 (1998), $67-87$.

[4] Cavaterra, C. and M. Grasselli: On an inverse problem for a model of linear viscoelastic Kirchhoff plate. J. Int. Equ. Appl. 9 (1997)3, 179 - 218.

[5] Colombo, F. and A. Lorenzi: Identification of time- and space-dependent relaxation kernels for materials with memory related to cylindrical domains. Part I: J. Math. Anal. Appl. 213 (1997), 32 - 62. 
[6] Colombo, F. and A. Lorenzi: Identification of time- and space-dependent relaxation kernels for materials with memory related to cylindrical domains. Part II: J. Math. Anal. Appl. 213 (1997), 63 - 90.

[7] Colombo F. and A. Lorenzi: An identification problem related to parabolic integrodifferential equations with non commuting spatial operators. J. Inv. Ill-Posed Probl. 8 (2000), $505-540$.

[8] Courant, R. and D. Hilbert: Methods of Mathematical Physics, Vol. I. New York: John Wiley \& Sons 1989.

[9] Fattorini, H. O.: Second Order Linear Differential Equations in Banach Spaces. Amsterdam: Elsevier Sci. Publ. 1985.

[10] Grasselli, M.: Identifying relaxation kernels of linearly viscoelastic bodies. J. Inv. Ill-Posed Probl. 4 (1996), 391 - 407.

[11] Grasselli, M.: On an inverse problem for a linear hyperbolic integro-differential equation. Forum Math. 6 (1994), 83 - 110.

[12] Grasselli, M.: An identification problem for an abstract linear hyperbolic integrodifferential equation with applications. J. Math. Anal. Appl. 171 (1992), 27 60.

[13] Grasselli, M., Kabanikhin, S. I. and A. Lorenzi: An inverse integro-differential problem arising in Geophysics, Part I. Siberian Math. J. 33 (1992), 415 - 462.

[14] Grasselli, M., Kabanikhin, S. I. and A. Lorenzi: An inverse integro-differential problem arising in Geophysics, Part II. Nonlin. Anal.: Theory, Meth. \& Appl. 15 (1990), 283 - 298.

[15] Janno, J.: Inverse problems for determining monotone weakly singular relaxation kernels in viscoelasticity. Nonlin. Anal.: Theory, Meth. \& Appl. 41 (2000), $943-962$.

[16] Janno, J.: Identification of weakly singular relaxation kernels in three-dimensional viscoelasticity. J. Math. Anal. Appl. 262 (2001), 133 - 159.

[17] Janno, J. and L. von Wolfersdorf: Inverse problems for identification of memory kernels in viscoelasticity. Math. Meth. Appl. Sci. 20 (1997), 291 - 314.

[18] Janno, J. and L. von Wolfersdorf: Inverse problems for memory kernels by Laplace transform methods. Z. Anal. Anw. 19 (2000), 489 - 510.

[19] Janno, J. and L. von Wolfersdorf: An inverse problem for identification of a time- and space-dependent memory kernel in viscoelasticity. Inverse Problems 17 (2001), $13-24$.

[20] Lorenzi, A.: A multidimensional identification problem related to a hyperbolic integro-differential equation. Z. Anal. Anw. 18 (1999), 407 - 435.

[21] Lorenzi, A. and V. G. Yakhno: An identification problem related to an isotropic nonhomogeneous stratified viscoelastic body. J. Inv. Ill-Posed Probl. 5 (1997), $29-53$.

[22] Lorenzi, A. and E. Paparoni: Identification of two unknown coefficients in an integro-differential hyperbolic equation. J. Inv. Ill-Posed Probl. 1 (1993), 331 348. 
[23] Nečas, J.: Les méthodes directes en théorie des équations elliptiques. Paris: Masson 1967.

[24] Prüss, J.: Evolutionary Integral Equations and Applications. Berlin: Birkhäuser Verlag 1993.

[25] Weiss, G. F.: A representation formula for strongly continuous cosine families. Aequ. Math. 21 (1980), $251-256$.

[26] von Wolfersdorf, L.: On identification of memory kernels in linear viscoelasticity. Math. Nachr. 161 (1993), $203-217$.

Received 02.07.2001 